\title{
Anthracyclins increase free PUFAs and etherPEs with PUFAs as potential hallmarks of lipid peroxidation and ferroptosis
}

\author{
David Balgoma ${ }^{1,}{ }^{*}$, Fredrik Kullenberg ${ }^{2}$, Carlemi Calitz ${ }^{3}$, Maria Kopsida ${ }^{3}$, Femke Heindryckx ${ }^{3}$, \\ Hans Lennernäs ${ }^{2}$, and Mikael Hedeland ${ }^{1}$ \\ 1 Analytical Pharmaceutical Chemistry, Department of Medicinal Chemistry, Uppsala University, Uppsala, Sweden; \\ david.balgoma@ilk.uu.se (D.B), mikael.hedeland@ilk.uu.se (M.H.) \\ 2 Department of Pharmaceutical Biosciences, Uppsala University, Uppsala, Sweden; fred-rik.kullenberg@farmbio.uu.se (F.K.), \\ hans.lennernas@farmbio.uu.se (H.L.) \\ 3 Department of Medical Cell Biology, Uppsala University, Uppsala, Sweden; carlemi.calitz@mcb.uu.se (C.C.), \\ maria.kopsida@mcb.uu.se (M.K.), femke.heindryckx@mcb.uu.se (F.H.) \\ * Correspondence: david.balgoma@ilk.uu.se
}

\begin{abstract}
Metabolic and personalized interventions in cancer treatment require a better under-standing of the relationship between the induction of cell death and metabolism. Consequently, we treated three primary liver cancer cell lines with two anthracyclins (doxorubicin and idarubin) and studied the changes of the lipidome. We found that both anthracyclins in the three cell lines increased the levels of polyunsaturated fatty acids (PUFAs) and alkylacylglycerophosphoethano-lamines (etherPEs) with PUFAs. As PUFAs and alkylacylglycerophospholipids with PUFAs are fundamental in lipid peroxidation during ferroptotic cell death, our results suggests supplementa-tion with PUFAs and/or etherPEs with PUFAs as a potential general adjuvant of anthracyclins. In contrast, neither the markers of de novo lipogenesis nor cholesterol lipids presented the same trend in all cell lines and treatments. In agreement with previous research, this suggests that modulation of the metabolism of cholesterol could be considered a specific adjuvant of anthracyclins depend-ing on the type of tumor and the individual. Finally, we discuss the changes in the lipidome in re-lation to the endoplasmic reticulum stress and the sensitivity to anthracyclins of the different cells. In conclusion, our results suggest that the modulation of different lipid metabolic pathways may be considered for generalized and personalized metabochemotherapies.
\end{abstract}

Keywords: hepatocellular carcinoma, plasmenyl, plasmanyl, plasmalogen

\section{1.-Introduction}

All cancers are characterized by an inherent metabolic reprograming that promotes tumorigenesis by facilitating and enabling proliferation, metastasis, and resistance to therapies ${ }^{1,2}$. Therefore, metabolomics and lipidomics play key roles in unravelling the metabolic transformation in cancer $^{3}$ and cancer treatment ${ }^{4}$. Hepatocellular carcinoma (HCC) is one of the most common cancers worldwide and it is known to cause profound modifications in lipid metabolism ${ }^{5}$. Among others, the malignant transformation of hepatocytes dysregulates the de novo lipogenesis ${ }^{5}$, which is hallmarked by the up-regulation of triacylglycerides (TGs) with saturated and monounsaturated fatty acids ${ }^{6}$. This altered lipid metabolism is involved in rapid tumor growth and adaptation to the tumor microenvironment ${ }^{7}$.

Clinicians have used anthracyclins, such as doxorubicin (DOX) and idarubicin (IDA), as chemotherapeutic agents against HCC among other solid tumors for more than five decades. 
Anthracyclins intercalate into the nucleus and mitochondrial DNA and subsequently inhibit the synthesis of proteins and affect the redox state of the cell. Anthracyclins also act on the mitochondrial electron transport and convert oxygen into reactive oxygen species that may cause mitochondrial dysfunction, change the redox state, and induce lipid peroxidation ${ }^{8}$. However, the interplay between anthracyclins and the lipidome is not fully understood. Different anthracyclins establish different physicochemical interaction with lipid membranes depending on the lipid composition ${ }^{9,10}$. For example, IDA is more lipophilic than DOX (logP 1.9 vs $\log P 1.3$ ) and 7-8 times more potent in vitro ${ }^{9,11}$. Depending on the lipophilic and amphiphilic nature of the anthracyclins, the lipidome of the cell affects drug internalization, and, consequently, its effect ${ }^{9}$. For example, DOX-resistant MCF-7 cells present an enrichment of glycerophospholipids and cholesterol lipids ${ }^{12}$. The changes in the lipidome may affect the further internalization of the drug and the mechanism of action of the drug to induce cell death, in which endoplasmic reticulum (ER) stress plays a key role. This interplay among anthracyclin uptake, the lipidome, and ER stress may partially explain the different sensitivity to anthracyclins in both cancerous and nontransformed cell (e.g. cardiomyocytes). The sensitivity is conditioned by the mechanism of cell death induced by anthracyclins, which depends on the cell type and drug concentration ${ }^{13}$. Consequently, understanding this interplay anthracyclin/lipidome/cell death might open the possibility to: i) improved diagnosis by biomarkers of the tumor resistance to anthracyclins ${ }^{14,15}$, and ii) proposal of novel treatments that may potentiate and/or complement the metabolic transformations caused by anthracyclins to induce cell-death.

Currently there are few studies about the effect of DOX on the lipidome of cancer cells and even less on the effect of other anthracyclins. For example, no lipidomics studies with IDA have been reported (Web of Science, December 2020). In addition, different studies have reported that lipidic modulation affects ER stress, cell death, and cardiotoxicity induced by anthracyclins ${ }^{2,16,17}$. Consequently, more research about the effect of anthracyclins on the cellular tumor lipidome is needed. In this study, we aimed to characterize the lipidic metabolic transformation of cancer cells after exposure to two different anthracyclins. We focused on finding and discussing common and differential changes in the lipidome to different anthracyclins and cell models in order to discuss the mechanism of cell death, the sensitivity to anthracyclins, and potential metabochemotherapies. Consequently, three different primary liver cancer cell lines (HepG2, Huh7, and SNU449) were treated with DOX or the more lipophilic and potent IDA 9,11. Subsequently, we investigated the regulation of the different lipids by their fold change, and found that both anthracyclins increased the levels of polyunsaturated fatty acids (PUFAs) and alkylacylglycerophosphoethanolamines (etherPEs) with PUFAs in all cell lines (Scheme 1). In the light of previous research, these lipids are involved in programmed cell death by ferroptosis ${ }^{18}$. The changes in cholesterol lipids were cell-type specific, as they increased in Huh7 and SNU449 cells, but did not present a clear trend in HepG2. Similarly, the glycerolipids of saturated and monounsaturated fatty acids did not present a consistent trend for both treatments in both cell lines. These lipids are of interest because they are markers of the liver-X-receptor (LXRs) and sterol regulatory element building proteins (SREBPs) pathways for de novo lipogenesis ${ }^{6}$. Finally, we discussed the relationship between the lipidome, ER stress, and the sensitivity to anthracyclins. From a broad perspective, our results suggest the possibility of using PUFAs and/or etherPEs with PUFAs as general adjuvant of anthracyclins to build new metabochemotherapy treatments. 


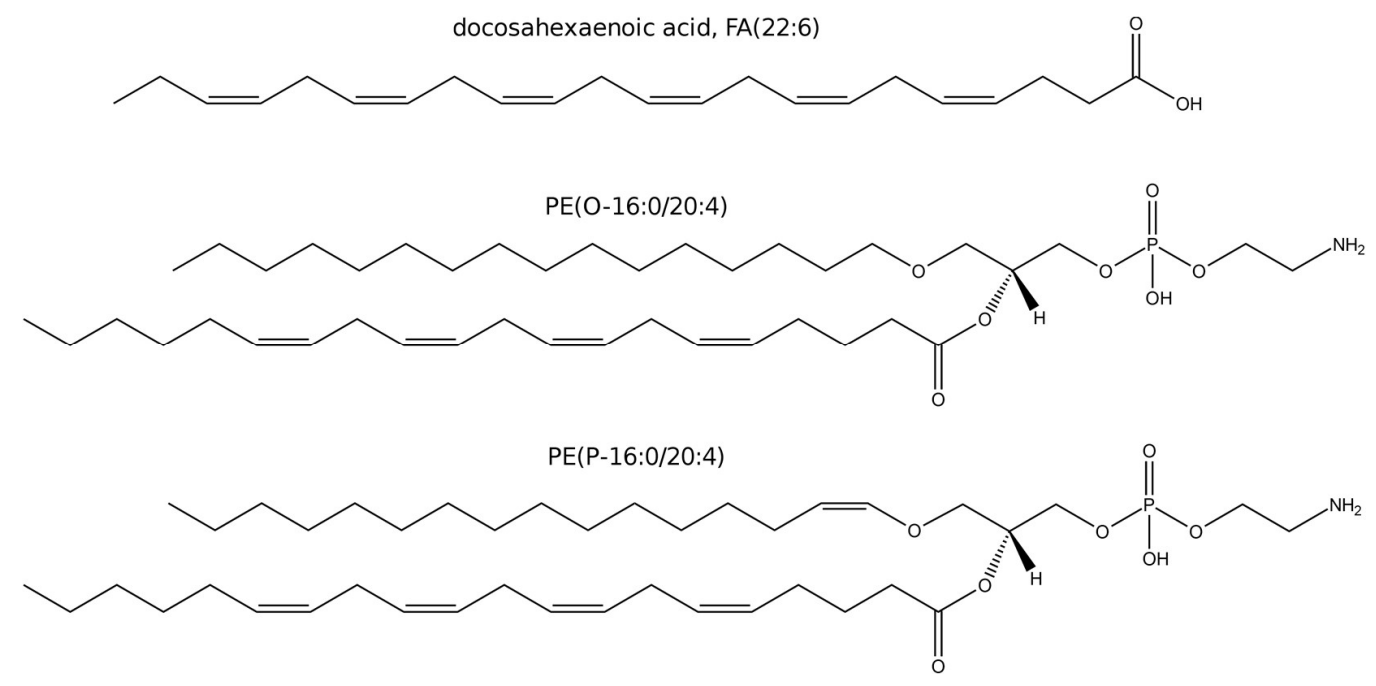

Scheme 1. Examples of a PUFA, FA(22:6); and two ether glycerophospholipids: a plasmanyl gly-cerophospholipid of ethanolamine, $\mathrm{PE}(\mathrm{O}-16: 0 / 20: 4)$; a plasmenyl glycerophospholipid of ethanolamine, $\mathrm{PE}(\mathrm{P}-16: 0 / 20: 4)$.

\section{Materials and Methods}

\subsection{Cell culture and treatment}

Three cell lines (HepG2, ATCC ${ }^{\circledR}$ HB-8065 $5^{\mathrm{TM}}$, SNU 449 ATCC ${ }^{\circledR}$ CRL-2234 ${ }^{\mathrm{TM}}$, and Huh7 from Dilruba Ahmed, Karolinska Institute, Sweden) were cultured at $37^{\circ} \mathrm{C}$ with $5 \% \mathrm{CO} 2$ and $95 \%$ humidity within a $\mathrm{CO} 2$ incubator. HepG2 and Huh7 were cultured in DMEM supplemented with $1 \%$ antibiotic antimycotic solution and 10\% FBS (cell culture media + FBS: CCMFed). SNU449 was cultured in RPMI medium supplemented with $1 \%$ antibiotic antimycotic solution and 10\% FBS (cell culture media + FBS: CCMFed). Standard culture medium without supplemented FBS was used during starvation (CCMSM). Misidentification of all cell lines was checked at the Register of Misidentified Cell Lines ${ }^{19}$. For authentication, extracted DNA from all three cell lines were sent to Eurofins Genomics (Ebersberg, Germany) for cell line authentication using DNA and short tandem repeat-profiles. Mycoplasma contamination was also tested.

Cells were seeded at a density of $4 \cdot 10^{6}$ cells per T75 flask $\left(75 \mathrm{~cm}^{2}, 60 \mathrm{ml}\right)$, and allowed to attach overnight. Prior to treatment, CCMFed was removed and the cells washed with $10 \mathrm{~mL}$ of PBS. To allow stabilization of the cell cycle, $15 \mathrm{ml}$ CCMSM was added to each flask containing cells 2 hours prior treatment. Cells were treated for 48 hours with either DOX or IDA in the form of a stock solution in DMSO containing $100 \mathrm{mM}$ anthracycline to achieve the concentrations in Table 1. For the respective controls, the amount of DMSO was matched as for the anthracyclin treatment (vehicle controls). In all cases, the percentage of DMSO was between $0.03 \%$ and $0.0005 \%$, which was at least 30 times below the limit at which DMSO presented cytotoxicity in our previous experiments ( $1 \%$ of DMSO, Kullenberg et al., submitted $\left.{ }^{20}\right)$. The exposure concentration of the anthracyclins was chosen to have cell mortality higher than $50 \%$ at $48 \mathrm{~h}$ of treatment. After 48 hours, the cells were washed twice with $5 \mathrm{ml}$ phosphate-buffered saline (PBS). A volume of $5 \mathrm{ml}$ PBS was added to each flask and the cells gently scraped using a cell scraper (TPP). The resulting cell suspension was collected and the cells counted using a TC20 ${ }^{\mathrm{TM}}$ Automated cell 
counter and counting slides (Bio Rad). The cell suspension was subsequently centrifuged at $140 \mathrm{~g}$ for 5 minutes, the supernatant removed and the pellet was resuspended in $250 \mu \mathrm{l}$ ice-cold MilliQ water and kept at -80 ㄷ C until analysis.

Table 1. Concentration in cell culture media of DOX and IDA treatments by cell type

\begin{tabular}{lll}
\hline Cell line & DOX concentration $(\mu \mathrm{M})$ & IDA concentration $(\mu \mathrm{M})$ \\
\hline HepG2 & 0.5 & 0.1 \\
Huh7 & 5 & 0.1 \\
SNU449 & 30 & 1 \\
\hline
\end{tabular}

For ER stress imaging and measurement, cells were fixed for 10 minutes in $4 \%$ paraformaldehyde and stored at $4^{\circ} \mathrm{C}$. Paraformaldehyde fixed cells were washed with tris-buffer saline (TBS) and antigen retrieval was done at $95^{\circ} \mathrm{C}$ in sodium citrate buffer for 45 minutes. Blocking was followed by an overnight incubation at $4^{\circ} \mathrm{C}$ with primer antibodies. 40-minute incubation was used for the secondary antibody (Rabbit anti-mouse Alexa Fluor-488 or donkey anti-rabbit Alexa Fluor-633) and cell nuclei were stained with Hoechst for 5 minutes. Images were taken using an inverted confocal microscope (LSM 700, Zeiss) using Plan-Apochromat 20x objectives and the Zen 2009 software (Zeiss). The different channels of immunofluorescent images were merged using ImageJ software. Quantifications were done blindly with ImageJ software by conversion to binary images for each channel and automated detection of staining on thresholded images.

\subsection{Lipidomics analysis and data pre-treatment}

After randomization of the order of sample preparation, $100 \mu \mathrm{L}$ of acetonitrile/isopropanol 50:50 were added to $10 \mu \mathrm{L}$ of sample. Proteins were precipitated overnight at $-20{ }^{\circ} \mathrm{C}$ and the supernatant was recovered after centrifugation ( $18000 \mathrm{RCF}, 10 \mathrm{~min}$ ). A quality control sample was built by pooling $10 \mu \mathrm{L}$ from every extract. The samples were analyzed as in Balgoma et al. ${ }^{21}$. The samples were injected in a randomized way on an Acquity UPLC hyphenated to a Synapt G2 Q-ToF (Waters) with electrospray ionization. The samples were analyzed in both positive and negative modes. The quality control pool was injected every five injections of samples. Lipids were identified as in Balgoma et al. ${ }^{21}$ by the $\mathrm{m} / \mathrm{z}$ of their adducts and their patterns of fragmentation. Due to the number of lipids, only the key species are represented in the main text. We detected 451 species of lipids peaks corresponding to free fatty acids (FA), lysophosphatidylcholine (LPC), phosphatidylcholine (PC), alkylacylglycerophospholipids of choline (etherPC), lysophosphatidylethanolamine (LPE), phosphatidylethanolamine (PE), etherPE, phosphatidylglycerol (PG), lysophosphatidylinositol (LPI), phosphatidylinositol (PI), lysophosphatidylserine (LPS), phosphatidylserine (PS), diacylglycerols (DG), TG, free sterols, cholesteryl esters (CE), ceramides (Cer) and sphingomyelin (SM).

\subsection{Data analysis}

We addressed the concerns about using p-values and "statistical significant" discoveries exposed by researchers, statisticians, and the American Statistical Association ${ }^{22-24}$. Consequently, we analyzed the changes in the lipidome by using fold changes (with confidence intervals) between the treatments and the control ${ }^{25}$. 
The cell size was quantified by the weighted mean of diameter of cells. The variability was calculated by the standard error of the mean of the three replicates.

Regarding lipidomics data, the areas of the peaks were normalized by the number of cells extracted. The changes in the lipidome were studied by the relative changes (increase/decrease) between the cells treated with anthracyclins and the cells treated with their respective controls (vehicle). These relative changes were quantified by the $\log$ (fold change), i.e. the natural logarithm of the ratio of the average of the normalized signal of the treated group divided by the average of the normalized signal of the control group (vehicle). The confidence interval of the $\log$ (fold change) was determined by the 10,000 bootstrap resampling simulations. The limits of the intervals of confidence were selected by the percentiles $2.5 \%$ and $97.5 \%$ of the simulations; the central measurement was characterized by the mean of the simulations. The values under the limit of detection (left-censored) were imputed by fitting the peak areas of a lipid to a normal distribution and random sampling of this distribution below the minimum detected signal. Fold changes with more than one value under the limit of detection in any group were discarded.

\subsection{Software and graphical material}

Waters' .Raw data files were transformed into .CDF format by Databride (Masslynx 4.1). Mass spectrometric data were pretreated with packages mzR 2.22.0 and XCMS 3.10.2 in R 4.0.3 "BunnyWunnies Freak Out". Graphical material was generated with R (packages forestplot 1.10 and ggplot2 3.3.2) and processed with Inskape 1.0.1 and Gimp 2.10.22. Microscopy images were obtained in Zen 2009 software (Zeiss) and further quantified and exported using ImageJ software.

\section{Results}

\subsection{Number and size of the recovered cells}

As expected, DOX and IDA induced a strong reduction in cell number when compared with the vehicle control. The respective vehicle controls did not induce cell death nor proliferation (Figure 1). In all cases, anthracyclins induced a reduction in cell number higher than $50 \%$, when compared with the vehicle.
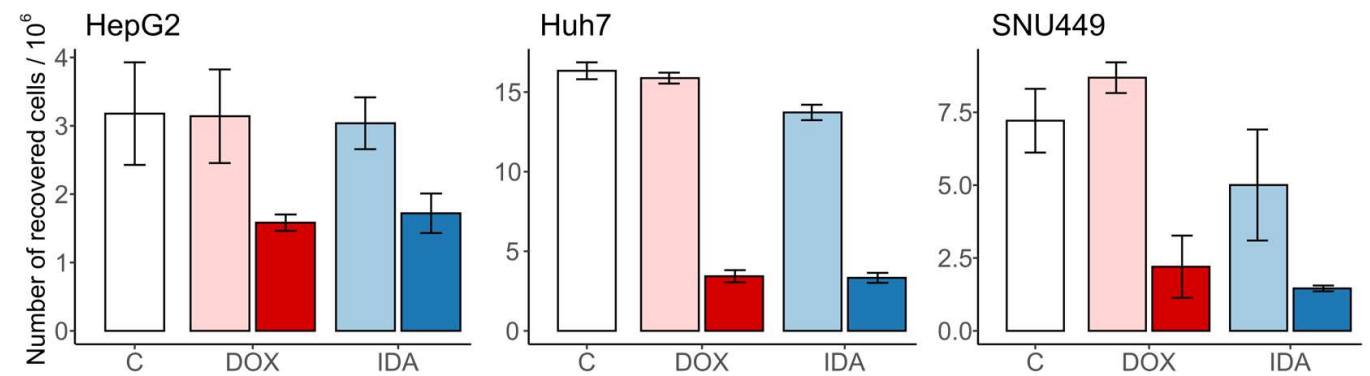

Figure 1. Number of recovered cells after treatment with anthracyclins. The untreated control (C) is in white, the vehicles of DOX and IDA are in light red and blue, respectively. The treatments of DOX and IDA are in dark red and blue, respectively. Whiskers represent the interval for the standard error of the mean.

Regarding the cell size, the vehicle controls did not change the diameter of the cells when compared with the untreated control (Figure 2). The treatment with anthracyclins did not change the cell volume for 
HepG2 nor Huh7. In contrast, we observed an increased cell diameter in DOX and IDA treated SNU449cells (Figure 2).
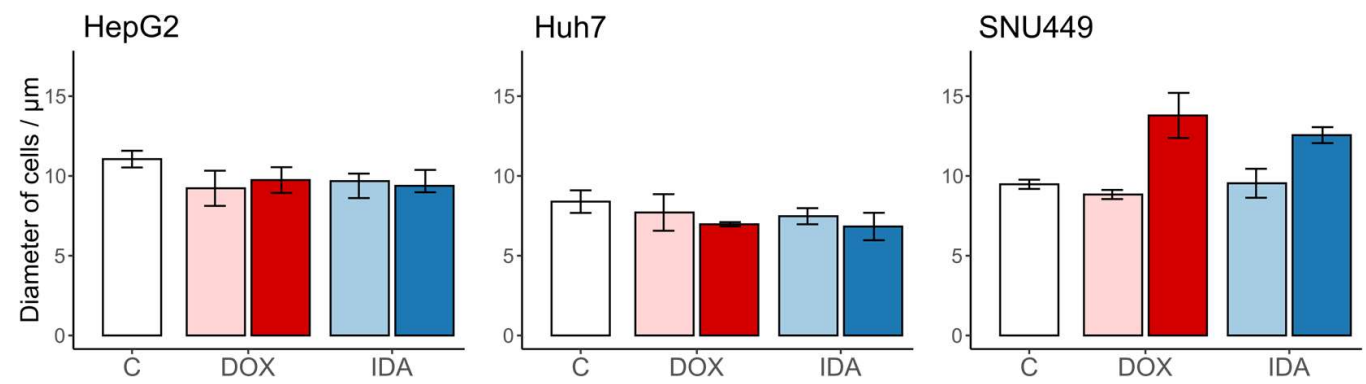

Figure 2. Size of the recovered cells (diameter, $\mu \mathrm{m}$ ) after treatment with anthracyclins. The untreated control (C) is in white, the vehicles of DOX and IDA are in light red and blue, respectively. The treatments of DOX and IDA are in dark red and blue, respectively. Whiskers represent the interval for the standard error of the mean.

\subsection{Anthracyclins increase etherPEs in all cell types}

To estimate the regulation of the total amount of lipids per family, we summed the normalized signal of every lipid family and calculated the logarithm of the fold change with respect to the vehicle-treated cells (Figure 3).

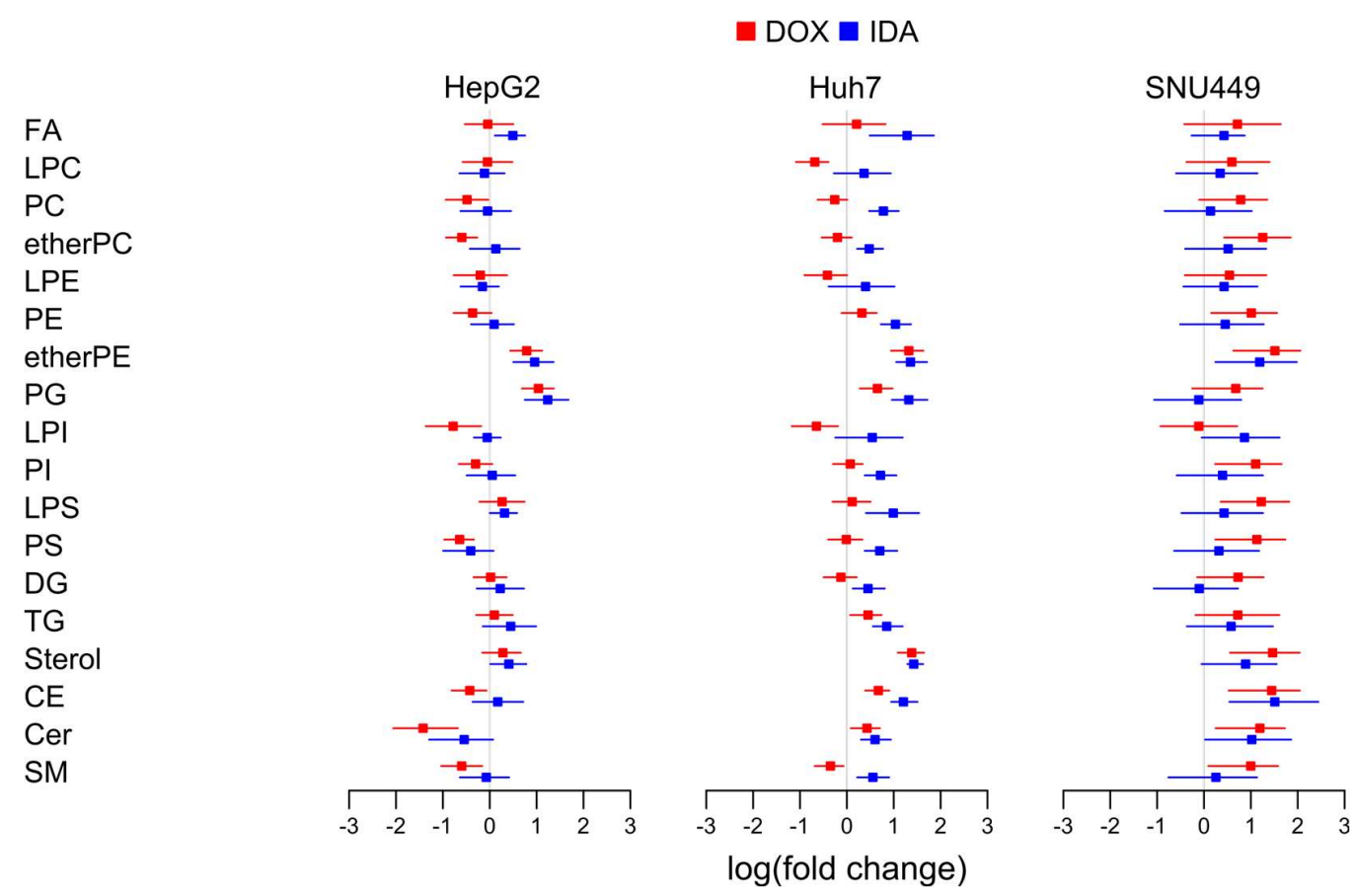

Figure 3. Logarithm of the fold change of the eighteen families of lipids in each cell line. We summed the signal of the lipids per type of cell, treatment, and replicate

In general, the different families of lipids presented no clear trend for all lipids for the three cell types. Regarding the ensemble of the lipid families for every cell type, the lipidome showed a trend to increase in SNU449, especially with DOX (Figure 3). 
Regarding common trends for both treatments and the three cell lines, we observed an increase of etherPEs. While most families of lipids increased in SNU449, the fold change of etherPEs was the highest, together with cholesterol lipids. This behavior points to a common and general metabolic effect of anthracyclins in etherPEs beyond the specificities of the metabolism of every cell line.

Regarding disparate trends for the different cell lines, cholesterol lipids (sterols and CEs) presented a trend to increase in Huh7 and SNU449, but not in HepG2. PGs presented a trend to increase in HepG2 and Huh7, but not a clear trend in SNU449. PSs and the sphingolipids (Cer and SM) presented a trend to decrease in HepG2, no clear trend in Huh7, and a trend to increase in SNU449. As highlighted before, the increase in these lipids in SNU449 was in a context of general increase in lipids in this cell line. Furthermore, Huh7 treated with IDA presented a general trend to increase.

\subsection{Anthracyclins increase all etherPEs with PUFAs, but not etherPC}

To study which lipid pathways were responsible for the upregulation of etherPEs in Figure 3, we represented the fold change of the individual species of alkylacylglycerophospholipids (etherGLs) and their fatty acid composition (Figure 4).

We only detected one species of etherPC, PC(0-16:0/18:1). This species did not present a clear trend for all cells and treatments (Figure 4). Regarding etherPEs, we detected six different species and all contained PUFAs. They corresponded to plasmanyl (ether bond) or plasmenyl (vinyl ether bond, Scheme 1), but their mass spectrometric characterization did not allow to distinguish these possibilities from each other. The fold change in etherPEs presented a common trend to increase in the three cell lines with both anthracyclins. We conclude that the upregulation of etherPEs with PUFAs (but not all etherGLs) was a general metabolic trait of anthracyclins.

$\mathrm{PC}(\mathrm{O}-16: 0 / 18: 1)$

$\mathrm{PE}(\mathrm{O}-16: 1 / 20: 4)$

$\mathrm{PE}(\mathrm{O}-16: 1 / 20: 5)$

$\mathrm{PE}(\mathrm{O}-18: 2 / 20: 3)$

$\mathrm{PE}(\mathrm{O}-18: 1 / 20: 4)$

$\mathrm{PE}(\mathrm{O}-38: 6)$

$\mathrm{PE}(\mathrm{O}-18: 2 / 20: 4)$ $\square$ DOX $\square$ IDA

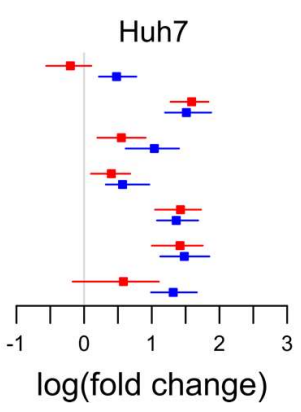

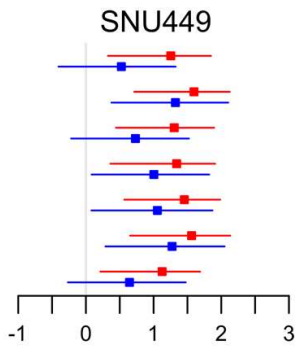

(2)

Figure 4. Logarithm of the fold change of the etherGLs. For etherPEs, the structure could also correspond to plasmenyls (e.g. PE(P-16:0/20:4) for PE(O-16:1/20:4))

\subsection{Anthracyclins increase free PUFAs}

To study if the availability of free PUFAs may partially control the increase of etherPEs with PUFAs, we investigated the fold change of FAs.

Despite the different regulation of different fatty acids, $F A(20: 4)$ and $F A(20: 5)$ showed a clear trend to increase in all cell lines treated with either DOX or IDA (Figure 5). Other PUFAs, such as FA(20:3) and 
$\mathrm{FA}(22: 6)$ also presented a trend to increase, but to a lower degree, especially in SNU449. This behavior suggests that free PUFAs are more available in all anthracyclin-treated cells.

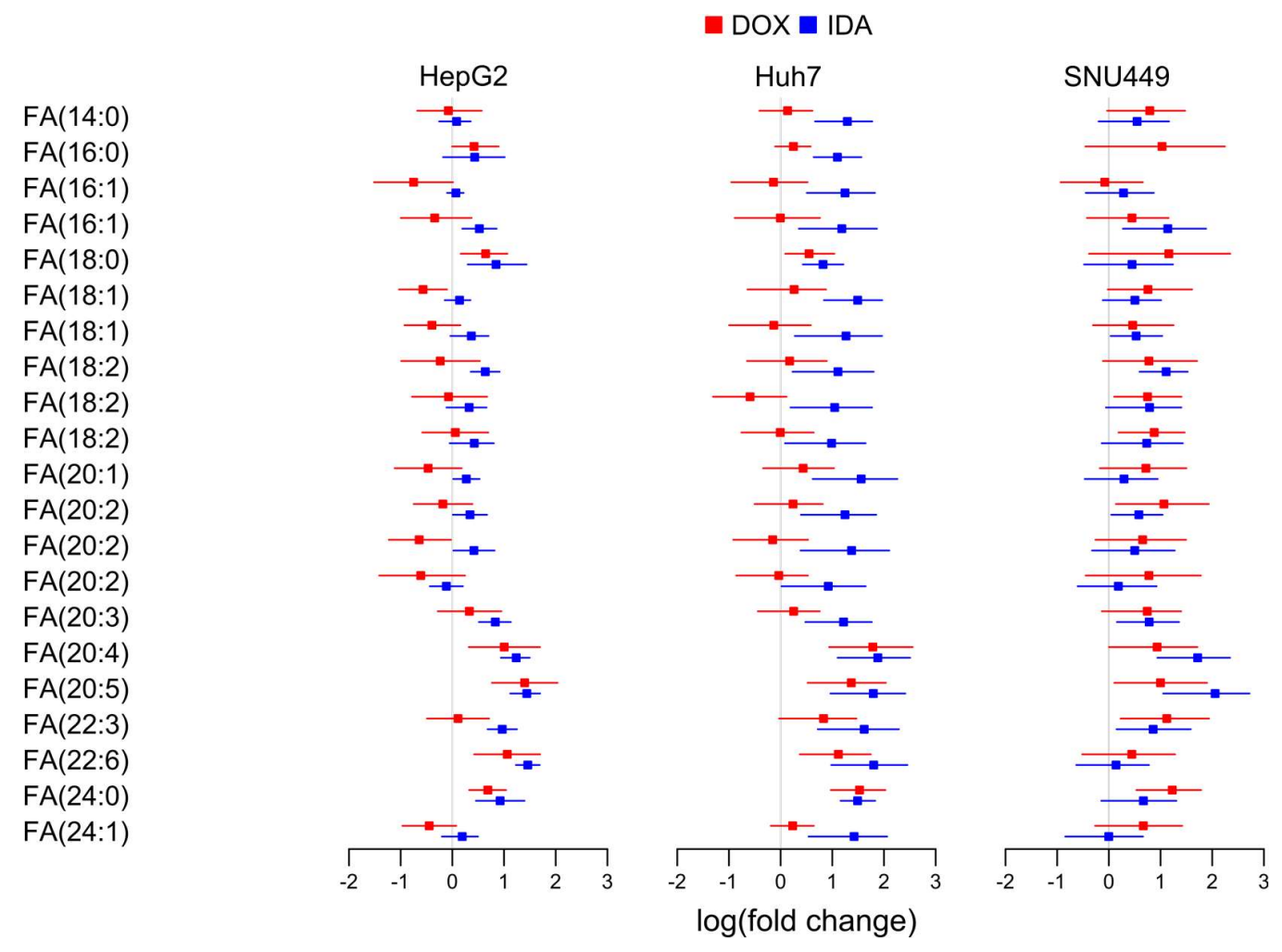

Figure 5. Logarithm of the fold change of free fatty acids (FAs) in each cell line.

\subsection{Anthracyclins increase other glycerolipids with PUFAs}

Radiolabeling experiments have shown that the glycerophospholipids of choline and ethanolamine contain most of PUFAs ${ }^{26}$. Consequently, we studied the levels of glycerolipids with PUFAs to investigate if a net release of PUFAs would explain the increase of free PUFAs. We observed that different molecular species presented different regulation (Figure 6), which might be explained by the different substrate selectivity in the enzymes involved in the liberation and incorporation of PUFAs into glycerolipids ${ }^{27}$. However, the major species tended to increase in all cells with all treatments. This was the case of $P C(16: 0 / 20: 4), \quad P C(18: 1 / 20: 4), \quad P E(18: 0 / 20: 4), \quad P I(18: 0 / 20: 4), \quad P C(16: 0 / 20: 5), \quad P C(18: 0 / 20: 5), \quad$ and $\mathrm{PC}(18: 1 / 20: 5)$ (Figure 6). Taken together, these upregulations suggest that the increase of free PUFAs (Figure 5) was not due to a net-release of PUFAs from glycerolipids.

Interestingly, $\mathrm{FA}(22: 6)$ (Scheme 1) might present different regulation than other PUFAs, as it is produced from $F A(24: 6)$ by peroxisomal $\beta$-oxidation ${ }^{28}$. The major species of glycerolipids with $F A(22: 6)$ in $P C$ and PE did not present a clear trend to decrease following anthracyclin treatment in any of the cell lines (Figure 6). Some minor species, such as PG(22:6/22:6) and TG(18:0/18:1/22:6) presented a trend to increase in treated HepG2 and Huh7. Nevertheless, these species did not present a clear increase in SNU449. In summary, the data do not suggest a general decrease for all cell lines of neither free nor esterified FA(22:6) (Figures 5 and 6). 

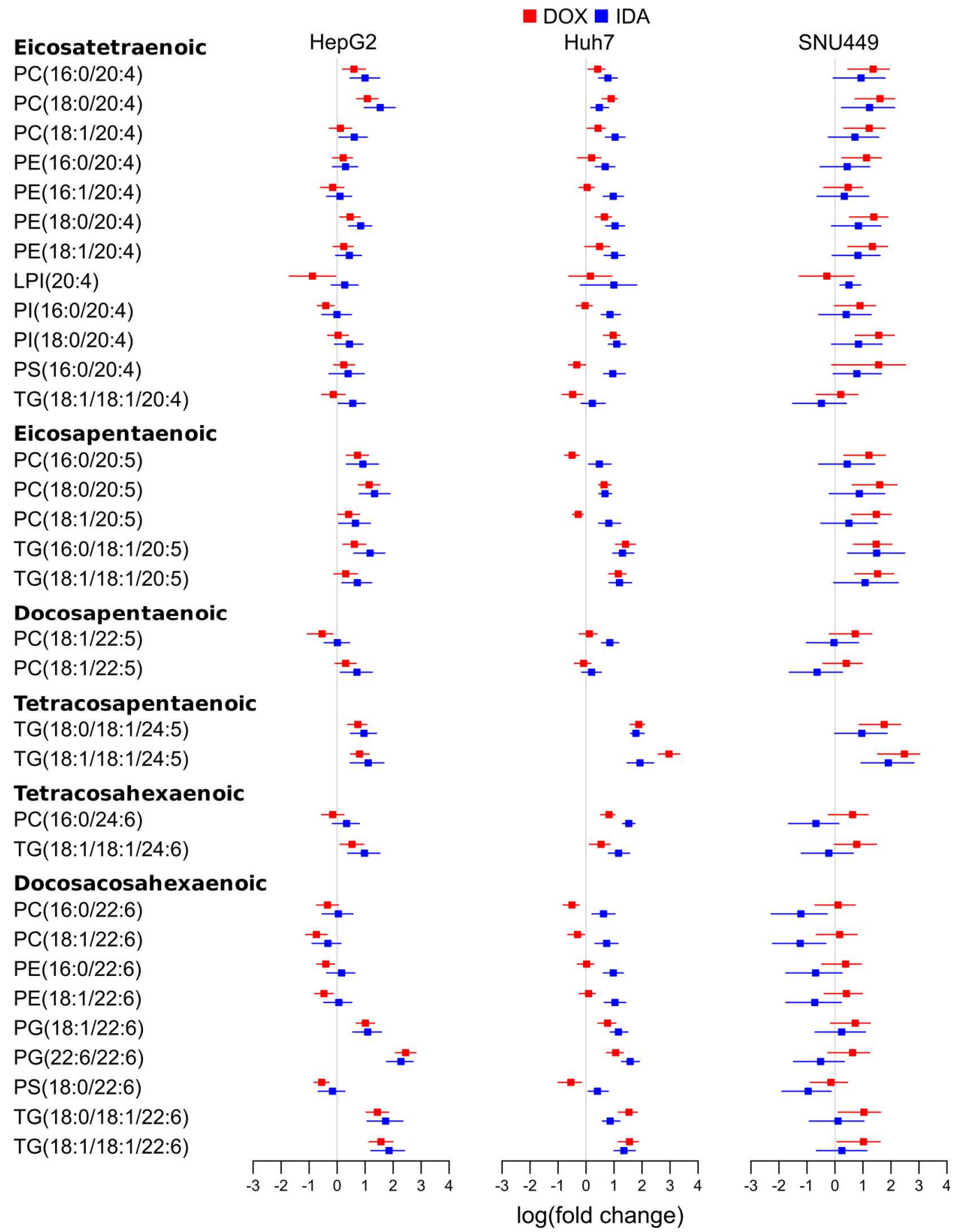

Figure 6. Logarithm of the fold change of selected glycerolipids with PUFAs detected in each cell line.

\subsection{Regulation of glycerolipids with saturated and monounsaturated fatty acids}

Glycerolipids with FA(16:0), FA(16:1), FA(18:0) and FA(18:1) are markers of LXR and SREBP pathways for de novo lipogenesis ${ }^{6}$. To evaluate if anthracyclins affect the de novo lipogenesis in the three cell lines, we studied the changes of these lipid species (Figure 7). 

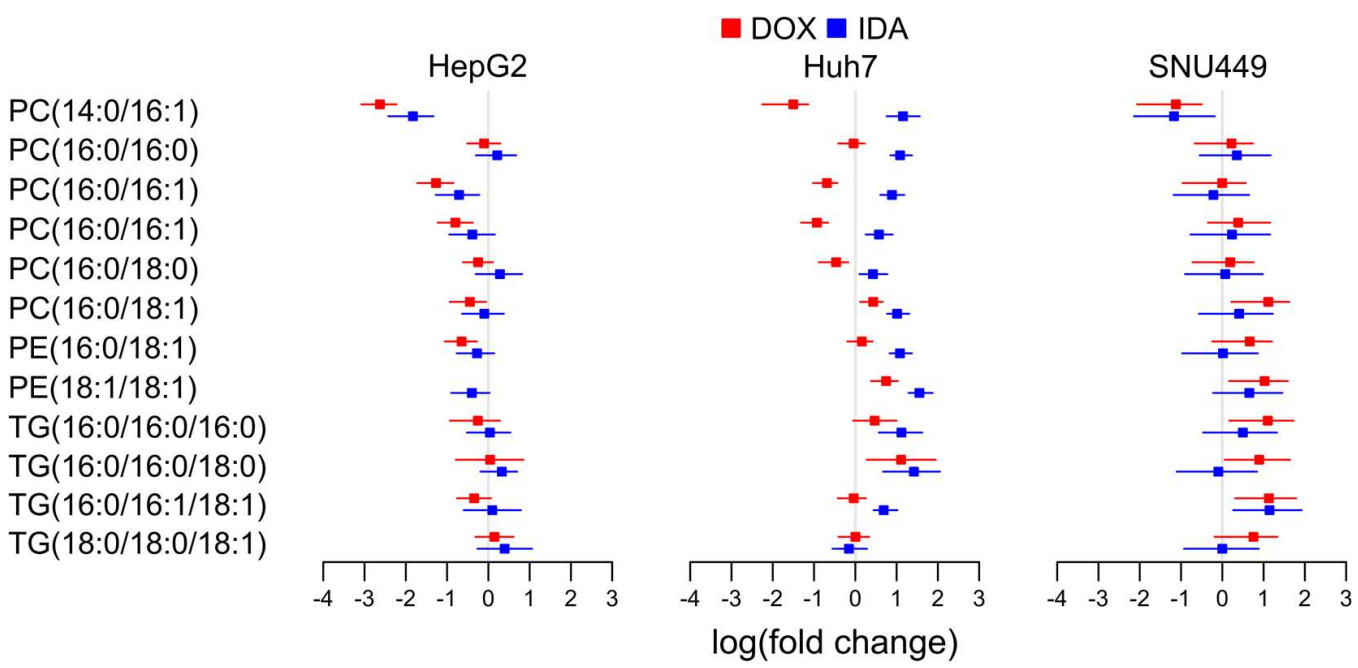

Figure 7. Logarithm of the fold change of selected glycerolipids with saturated and monounsaturated fatty acids in each cell line.

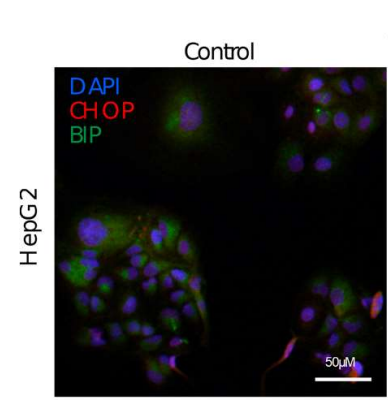

A
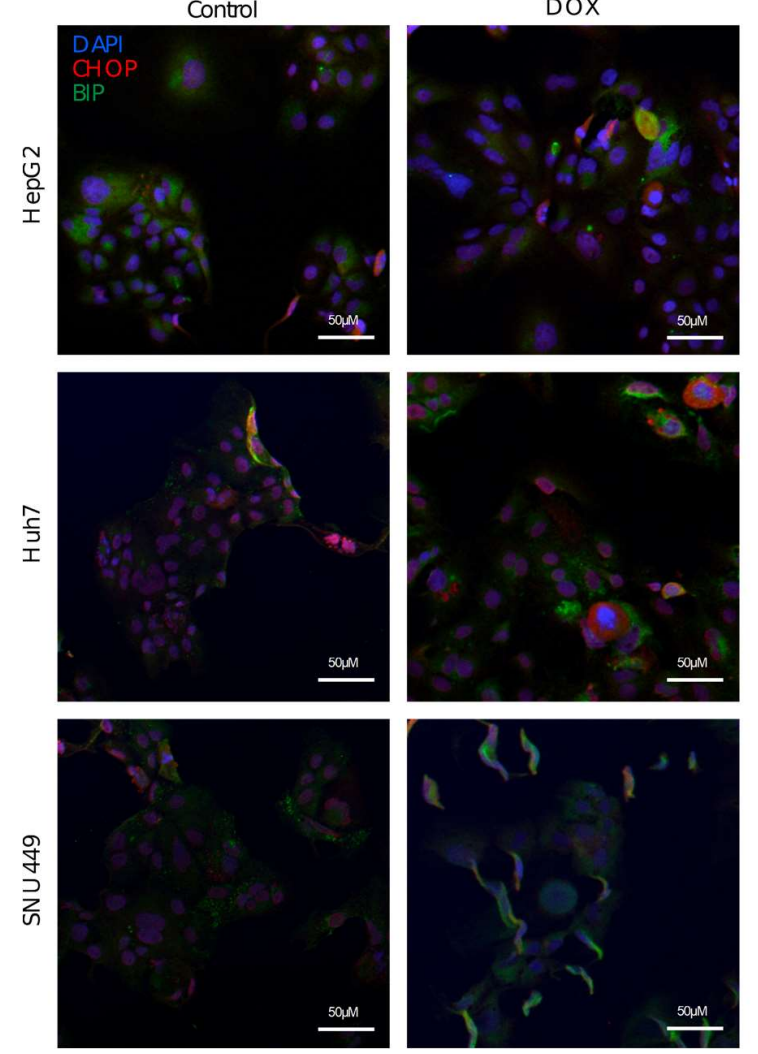

B
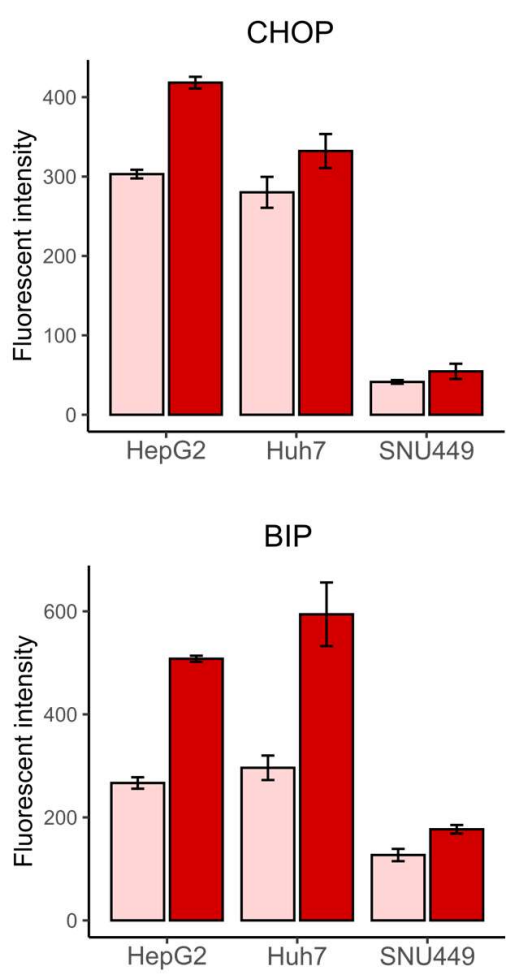

Figure 8. DOX treatment increase markers of ER stress. A) Representative images of HepG2, Huh7, and SNU449 tumor cells stained with antibodies against DAPI (blue), CHOP (red), and BIP (green). B) Quantification of the levels of $\mathrm{CHOP}$ and BIP in the cells with vehicle controls (light red) and treatments with DOX (dark red). 
Despite the specific decrease in species like PC(14:0/16:1) in HepG2, the general overview of the main glycerolipid species with saturated and monounsaturated fatty acids did not show any clear trend. Only in Huh7 treated with IDA there was an increase in these species, except for TG(18:0/18:0/18:1). This suggests that anthracyclins do not affect LXRs nor SREBPs de novo lipogenesis in a general way, but it could depend on the cell type-treatment combination.

\subsection{DOX increases markers of ER stress}

To study ER stress, we determined the levels of DNA damage-inducible transcript 3 (a.k.a. C/EBP homologous protein, CHOP) and binding immunoglobulin protein (BIP) through immunocytochemistry (Figure 8). These two markers increased after DOX treatment, which confirms that anthracyclins induce ER stress. The increase was stronger for HepG2 and Huh7, than for SNU449.

\section{Discussion}

Here we present that anthracyclins elicit the upregulation of PUFAs and etherPEs with PUFAs in different primary liver cancer cells. By using DOX and IDA in three different cell lines, we present new evidence that this is a general effect of anthracyclins on the metabolism of cancer cells. We also present evidence that the regulation of cholesterol lipids and de novo lipogenesis by anthracyclins is cell-type dependent for three different cell lines of primary liver cancer. As etherPEs with PUFAs are involved in programmed cell death by ferroptosis ${ }^{18}$ and statins have been suggested as anthracyclin adjuvants ${ }^{29}$, this metabolic characterization may help to design general and personalized metabochemotherapies for improved cancer treatments.

\subsection{Comparison with other lipidomics studies.}

We did not observe a general change of lipids in HepG2 and Huh7, except for the increase of PUFAs, etherPEs with PUFAs, and PGs (Figure 3). This observation agrees with the recent study by Newell et al. in 2020, which does not report strong changes in the lipid families in breast cancer cells (MDA-MB-231 and MCF-7) treated with DOX in combination with fatty acids ${ }^{30}$. They did not include etherPEs nor PGs in their study, so, as new result, we observed that both anthracyclins upregulated these families of glycerophospholipids in HepG2 and Huh7 (PUFAs and etherPEs with PUFAs increased in SNU449 as well, Figure 3).

In contrast, Achkar et al. in 2020 found a general downregulation of most metabolites and lipids (including etherPEs with PUFAs) in egg-developed tumors (MDA-MB-231) treated with DOX ${ }^{31}$. This finding is in apparent contradiction to our results and those reported by Newel et al. in $2020^{30}$. Nevertheless, the study of Achkar et al. in 2020 about DOX-treated solid tumors contained alive, damaged, and dead cell debris $^{31}$. We investigated the change in the lipidome of viable cells and normalized the amount of lipids to the number of cells. The differences in the cell subpopulation and the normalization may explain this observed discrepancy.

In conclusion, our study complements previous studies as we observed that PUFAs and etherPEs with PUFAs were increased in general after the treatment with anthracyclins in three different cell lines. This 
observation suggests that the increase of these lipids is a general metabolic trait of anthracyclins in liver cancer cells.

\subsection{Regulation of the de novo lipogenesis of fatty acids, glycerolipids, and cholesterol.}

LXRs and SREBPs control the expression of the enzymes responsible for the de novo lipogenesis of fatty acids, glycerolipids, and cholesterol ${ }^{32}$. The increase in de novo lipogenesis of fatty acids and glycerolipids is characterized by the upregulation of glycerolipids with saturated and monounsaturated fatty acids ${ }^{6}$. In this context, we did not observe a general up or downregulation of these glycerolipids, which suggests that anthracyclins do not modify the de novo synthesis of fatty acids in general (Figure 7). Regarding the regulation of cholesterol, we observed a trend to upregulation of cholesterol lipids in Huh7 and SNU449 (Figure 3). However, this trend was not clear for HepG2. Consequently, our data do not suggest a general trend for the de novo lipogenesis of neither fatty acids nor cholesterol.

Previous studies also report disparate results regarding the regulation of LXR/SREPB pathways by anthracyclins. On the one hand, it has been reported that DOX slightly upregulates SREBPs in the kidney with disparate behavior of the enzymes responsible for the de novo synthesis of fatty acids and cholesterol $^{33}$. In cardiomyocytes, DOX also upregulates oxysterols and hence elicits LXR activation ${ }^{34}$. On the other hand, other studies report downregulation of 3-hydroxy-3-methyl-glutaryl-coenzyme A reductase (HMGCR) in epidermal and prostate cancer cell lines ${ }^{35}$. Furthermore, chemotherapy may modify the cholesterol efflux from the environment of the cell, which could affect the cholesterol content in many cellular compartments ${ }^{36}$.

Taken our results and previous research together, we conclude that the up or downregulation of the LXR/SREBP-mediated de novo lipogenesis of fatty acids, glycerolipids, and cholesterol was not a general effect of anthracyclins. Nevertheless, as we saw different regulation for three different lines of the same type of cancer, it could depend on the cell type, tumor, or even individual genotype and phenotype.

\subsection{Regulation of the increase of etherPEs with PUFAs and PUFAs by anthracyclins.}

The synthesis of etherGLs is initiated in the peroxisome and completed in the ER, where the activity of alcohol reductase FAR1 is the limiting step (Scheme 2) ${ }^{37-39}$. The amount of etherGLs is regulated by: i) the downregulation of FAR1 by the increase of etherGLs in the inner leaflet of the plasma membrane, and ii) the lysoplasmalogenase activity ${ }^{40}$. While we observed an upregulation of etherPEs with PUFAs in all cells and treatments (Figure 4), we detected an etherPC, $\mathrm{PC}(0-16: 0 / 18: 1)$, that did not present a consistent increase in all treatments and cells. This differential behavior suggests that the upregulation of etherPEs with PUFAs was independent of: i) a putative increase of the synthesis of 1-0-alkyldihydroxyacetone phosphate in the peroxisome (Scheme 2), or ii) a putative decrease in the lysoplasmalogenase activity. Free PUFAs increased in a general way. Consequently, it seems plausible that the availability of PUFAs was the metabolic regulation responsible for the increase of etherPEs with PUFAs. 


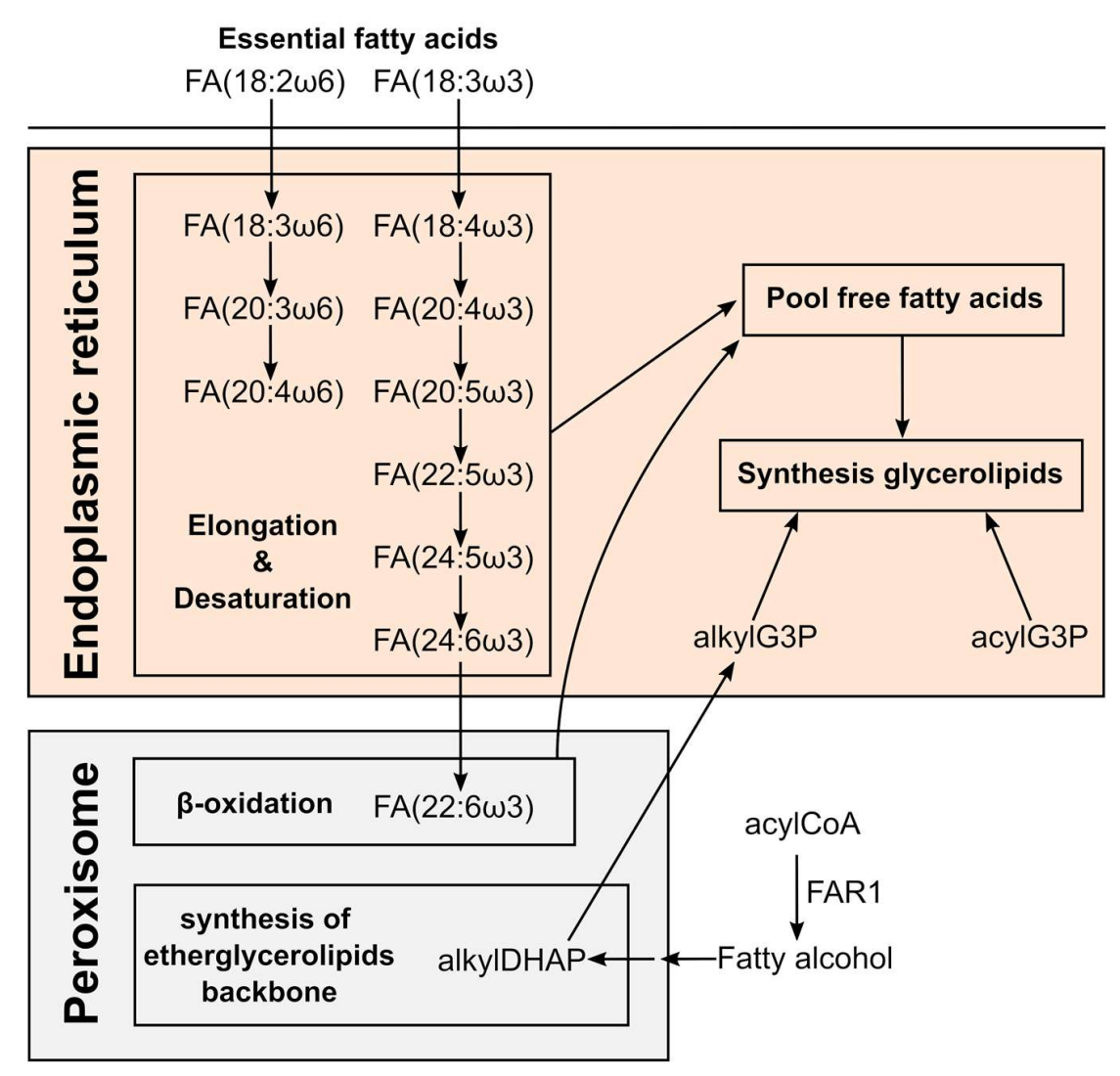

Scheme 2. Key molecules in the biosynthetic route of PUFAs and ether glycerolipids in different human subcellular compartments. Adapted from ${ }^{41-43}$.

The levels of free PUFAs could be increased by three different factors: i) the increase of their synthesis by elongation and desaturation of essential linoleic and linolenic acids ${ }^{42}$; ii) the balance between their liberation from and incorporation into glycerolipids $26,27,44,45$; and/or iii) the downregulation of their degradation by peroxisomal $\beta$-oxidation ${ }^{37}$. Consequently, we analyzed the levels of PUFAs in different glycerolipids to discuss the potential involvement of these three processes. On the one hand, the general trend to upregulation of $\mathrm{FA}(20: 4)$ and $\mathrm{FA}(20: 5)$ in glycerolipids (Figure 6) suggests that their net-release is not responsible for the increase of their free form. On the other hand, their degradation is initiated in the peroxisome by $\beta$-oxidation. Interestingly, docosahexaenoic acid, $\mathrm{FA}(22: 6)$, is not synthesized by elongation and desaturation in the ER, but by $\beta$-oxidation of $F A(24: 6)$ in the peroxisome (Scheme 2$)^{46}$. Consequently, the decrease of free and esterified FA(22:6) would be indicative of the downregulation of peroxisomal $\beta$-oxidation and, consequently, of the degradation of PUFAs. However, we did not observe a general downregulation of $F A(22: 6)$ neither free nor esterified. In fact, we rather observed a trend to increase in specific lipid species in HepG2 and Huh7 (Figure 6). The behavior of FA(22:6) suggests that neither the activity of peroxisomal $\beta$-oxidation nor the degradation of PUFAs were downregulated as a general characteristic of anthracyclins. 
We conclude that the observation of the ensemble of free PUFAs and glycerolipids with PUFAs suggests the upregulation of the elongation and desaturation of linoleic and linolenic acids as responsible for the increase of free PUFAs and etherPEs with PUFAs (Scheme 2).

\subsection{Role of PUFAS and etherPEs with PUFAs: potential hallmark of programmed cell death by ferroptosis.}

Two main lines of research have related PUFAs and cell-death induced by anthracyclins: i) PUFAs enhance the effect of DOX on cell death, and ii) DOX induces ferroptosis, in which PUFAs, etherPEs with PUFAs, and ER stress are involved.

For more than 25 years, it has been known that extracellularly added PUFAs enhance the sensitivity of tumor cell lines to DOX ${ }^{47,48}$. Among different PUFAs, FA(22:6) presents the highest enhancement of cytotoxicity $^{48}$. This effect has also been demonstrated in animal models ${ }^{30}$. However, the mechanism(s) of how PUFAs enhance anthracyclin-induced cell death is(are) not fully understood. Pioneering studies found that PUFA peroxidation might play a key role in boosting the oxidative stress in the tumor cell ${ }^{49}$. In this context, we observed that anthracyclins alone elicited the upregulation of PUFAs. Interestingly, supplementation of cells with PUFAs also enhances cell death by ferroptosis, the other line of research ${ }^{50}$. Considering that we observed a general increase of PUFAs and that DOX-induced cell death has been associated with ferroptosis ${ }^{51}$, it seems plausible that this line of research converges with ferroptosis research. Consequently, the enhancement of cell death induced by PUFAs might be explained by boosting lipid peroxidation in anthracyclin-induced ferroptosis ${ }^{52}$.

Ferroptosis, a term for a non-apoptotic cell death introduced in 2012, is characterized by the intracellular accumulation of lipid hydroperoxides ${ }^{50,53}$. Lipid peroxides yield aldehydes that react with intracellular and membrane proteins, compromising their function. To achieve lipid peroxidation, ferroptosis requires changes in a plethora of pathways, such as iron, glutaminolysis, and cholesterol and mevalonate pathways ${ }^{53}$. Furthermore, ferroptosis does not imply modifications in the morphology of the cell, except for the mitochondria ${ }^{54}$.

In the context of lipid peroxidation and ferroptosis, first, our analysis about the regulation of PUFAs indicates that their increase was due to the upregulation of the elongation and desaturation (Section 4.3, Scheme 2). In this process, $\triangle 6$-fatty acid desaturase 2 (FADS2) is necessary for the synthesis of PUFAs ${ }^{55}$. Interestingly, the knockdown of FADS2 presents attenuated lipid peroxidation in Huh7 cells ${ }^{56}$. Consequently, the upregulated elongation and desaturation to yield PUFAs (Section 4.3) is consistent with the increase in lipid peroxidation and ferroptosis.

Second, we found a general increase of etherPEs with PUFAs. It is known that: i) glycerophospholipids of ethanolamine with PUFAs are necessary for ferroptosis ${ }^{57}$; and ii) etherGLs with PUFAs present a critical contribution to ferroptosis ${ }^{18}$. It is plausible that the increase of etherPEs with PUFAs can be considered a hallmark of ferroptosis in primary liver cancer treated with anthracyclins. The reason why etherGLs with PUFAs are important in ferroptosis is not clear. Interestingly, they localize in the internal leaflet of the plasma membrane ${ }^{40}$, which suggests that lipid peroxidation in this region of the cell plays a key role in the cell death by ferroptosis. 
Finally, regarding the morphology, we found that HepG2 and Huh7 did not change their size upon anthracyclin treatment. However, SNU449 increased in size with both DOX and IDA. Furthermore, we observed an increase in PUFAs and etherPEs with PUFAs in SNU449, but in the context of a general increase of lipids (Figure 3). EtherPEs with PUFAs presented a slightly higher fold change than other lipids in SNU449 (Figure 3), but the overall change of the lipidome and the increase in size suggest that the enrichment of lipids with PUFAs was diluted in the membranes. Consequently, it seems plausible that lipid peroxidation in SNU449 was quenched.

Considering the discussion in the three previous paragraphs, we conclude that the changes in the lipidome and the cell size suggest that ferroptosis may drive the cell death for HepG2 and Huh7. However, other mechanisms apart from lipid peroxidation and ferroptosis may drive the cell death for SNU449. This suggestion is consistent with previous studies, as the mechanism of cell death depends on the concentration of anthracyclins and the cell type ${ }^{13}$.

\subsection{Interplay among the lipidome, anthracyclin uptake, ER stress, and sensitivity to anthracyclins.}

The metabolome, the lipidome, the proteome, and the transcriptome affect anthracyclin uptake and ER stres ${ }^{58,59}$. Anthracyclins themselves affect the 'omes, which further modifies the uptake of the drug and ER stress. The outcome of this crossed interplay is the different mechanisms of cell death and hence the sensitivity of a cell/tumor type to anthracyclins ${ }^{13}$. Of special interest in this interplay is the lipidome, as it conditions: i) drug uptake (drug-membrane interaction and membrane fluidity), ii) ER stress (ratio PC/PE, balance of PUFAs versus cholesterol and saturated lipids), and iii) the type of cell death (lipids with PUFAs in ferroptosis) (Scheme 3).

Regarding anthracyclin uptake, lipid membrane composition determines its fluidity and drug membrane permeation, as well as activation of drug carrier-mediated efflux mechanisms related to multidrug resistance ${ }^{9,60}$. We have found that the intracellular uptake ratio of DOX was one order of magnitude higher in HepG2 or Huh7 than in SNU449 (HepG2 > Huh7 >> SNU449, Kullenberg, et al., submitted $\left.{ }^{20}\right)$. Consequently, we speculate that the different response of the lipidome of the three cell lines (Figure 3) may play a role in the higher uptake of DOX by HepG2 and Huh7. Interestingly, PGs presented a remarkable increase in HepG2 and Huh7 with both anthracyclins (Figure 3). PGs are negatively charged, and interact in the membrane with positively charged DOX and IDA. Other lipids also affect the properties of the membranes regarding drug uptake: i) the levels of other negatively charged lipids (PIs, PSs); ii) zwitterionic lipids (PCs, PEs) ${ }^{10}$; and fluidity (cholesterol and saturation of fatty acids in the glycerolipids). Our study was limited to three cell lines, which prevents the discussion of these multiple changes. Nevertheless, in the light of previous research, we speculate that the strong enrichment of PGs in the membranes of HepG2 and Huh7 (Figure 3) may play a role in favoring anthracyclin uptake.

ER stress plays an essential role in apoptosis and ferroptosis ${ }^{61}$, as well as in promoting tumorigenesis in $\mathrm{HCC}^{62,63}$. In addition, ER stress mediates drug resistance to chemotherapeutic agents ${ }^{64}$. There is a crosstalk between ER stress pathways and the lipidome ${ }^{65}$. In this crosstalk, more than the composition in a specific type of lipid, it is the balance among different types of lipids what is associated with ER stress. For example, the imbalance between lipids with PUFAs versus saturated and cholesterol provokes ER stress $^{58,59}$. In this context, we observed that: i) membrane lipids with PUFAs increased in general in each 
of the three cell lines (Section 4.3), ii) membrane lipids with saturated and mono-unsaturated fatty acids did not present any clear trend (Section 4.2), and ii) cholesterol lipids increased in Huh7 and SNU449, but not in HepG2 (Figure 3). This ensemble of observations suggests a higher imbalance in HepG2 cells than in SNU449 (Scheme 3), which in the light of previous research partially explains the stronger increase of ER stress in HepG2 cell than in SNU449 (Figure 8). Many other factors beyond the scope of this study affect ER stress ${ }^{65}$, which may explain why Huh7 did not present a strong imbalance between lipids with PUFAs and sterols but presented a strong increase of ER stress (Figure 8B).

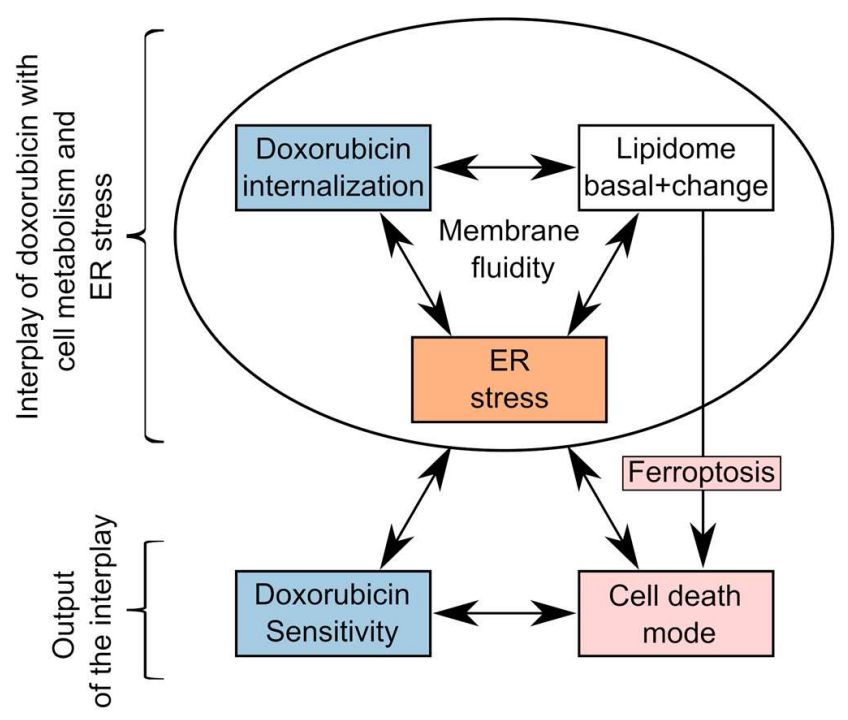

\begin{tabular}{|c|c|c|c|}
\hline & HepG2 & Huh7 & SNU449 \\
\hline PUFAs & $\uparrow$ & $\uparrow$ & $\uparrow$ \\
\hline \multicolumn{4}{|l|}{ vs } \\
\hline SFAs & $\leftrightarrow$ & $\leftrightarrow$ & $\leftrightarrow$ \\
\hline Chol. lipids & $\leftrightarrow$ & $\uparrow$ & $\uparrow$ \\
\hline ER stress & $\uparrow \uparrow$ & $\uparrow \uparrow$ & $\uparrow$ \\
\hline Cell size & $\leftrightarrow$ & $\leftrightarrow$ & $\uparrow$ \\
\hline $\begin{array}{c}\text { Global } \\
\text { lipidome }\end{array}$ & $\leftrightarrow$ & $\leftrightarrow$ & $\uparrow$ \\
\hline $\begin{array}{l}\text { Suggested } \\
\text { death mode }\end{array}$ & Ferr & Ferr & $\begin{array}{c}\text { Ferr and/or } \\
\text { other }\end{array}$ \\
\hline $\begin{array}{l}\text { Doxorubicin } \\
\text { sensitivity }\end{array}$ & $\uparrow \uparrow \uparrow$ & $\uparrow \uparrow$ & $\uparrow$ \\
\hline
\end{tabular}

Scheme 3. On the left, schematic interplay between DOX, the lipidome, and ER stress for a cell type, with the output of sensitivity to the drug and cell death mode. On the right, ensemble of qualitative trends of DOX treatment for the three cell lines: i) the balance between PUFAs lipids versus saturated fatty acids (SFAs) and cholesterol lipids (Chol. Lipids), ii) ER stress, iii) cell size and the global lipidome, iv) suggested death mode (Ferr for ferroptosis) and DOX sensitivity.

Considering the ensemble data about the lipidome, drug uptake, and ER stress (Scheme 3), the observed changes may contribute to explain why SNU449 is one of the most resistant cell lines to anthracyclins ${ }^{66}$. In comparison with HepG2 and Huh7, SNU449 presented: i) an increase in size (Figure 2), ii) a general increase in the lipidome (Figure 3), iii) a weaker increase in ER stress makers (Figure 8). Despite other factors (metabolome, proteome, transcriptome) also take part in the cell death, this differential behavior in the context of previous research suggests a key role of lipids in cell death. In this role, more than the change in one lipid or lipid family, it is the ensemble of lipids which seems involved in the resistance to anthracyclins.

In conclusion, as discussed in previous paragraphs, the sensitivity to anthracyclins can be related to a complex interplay among the lipidome, cellular uptake and intracellular disposition of drugs, ER stress, and the mechanism(s) of cell death. These factors depend on one another and on other factors beyond the scope of this study (transcriptome, proteome, metabolome). This interplay prevents the discussion of 
the effect of a lipid or a family of lipids in isolation. Despite these limitations, previous research give sense to the observed changes in the lipidome in relation to drug uptake, ER stress, and the sensitivity of the cells to anthracyclins for HepG2, Huh7, and SNU449.

\subsection{Perspectives in metabochemotherapy and anthracyclins}

It is known that PUFAs potentiate the effect of DOX in vitro and in animal models ${ }^{30,67}$. This effect might be general, as it would potentiate the lipid peroxidation in ferroptosis induced by anthracyclins. However, potentiating ferroptosis may increase the cardiotoxicity of anthracyclins ${ }^{68}$. Paradoxically, it has been reported that PUFAs may have a cardioprotective effect in anthracyclin chemotherapy ${ }^{69,70}$. This suggests that PUFAs may present a therapeutic window. In this window, PUFAs may potentiate anthracyclininduced ferroptosis in tumor cells, and in parallel minimizing cardiotoxicity. Similarly, the administration of anthracyclins in bilayers with etherPEs with PUFAs may also present a therapeutic window. Our results suggests that this would be the case for tumors with cells with lipid metabolism similar to HepG2 and Huh7. As discussed before, ferroptosis might not be the only suggested mechanism of death for SNU449. Nevertheless, we speculate that the treatment with PUFAs and/or etherPEs with PUFAs might tumors with cells like SNU449 into the path of lipid peroxidation and ferroptosis. In this perspective, PUFAs and etherPEs with PUFAs could be considered general adjuvants of anthracyclins.

Another suggested adjuvant of anthracyclins is the inhibition of cholesterol formation by statins ${ }^{71}$. Our results suggest that the effect of anthracyclins on cholesterol lipids was specific for each of the the three cell lines of the same type of tumor. The fact of having a different behavior for three cell lines of the same type of tumor indicates that the modulation of the cholesterol pathway would be of interest depending on both the cancer type and the patient. This agrees with previous literature, as statins present synergizing and protective effects in different models ${ }^{29,72-74}$. Furthermore, the adjuvant effect of statins is not clear in patients ${ }^{71}$. An explanation would be that the adjuvant effect of statins might depend on the patient pharmacokinetics, pharmacodynamics, and cholesterol metabolism. Consequently, while statins do not seem to be a general adjuvant of anthracyclins, it seems plausible that they could be used for specific tumors and in personalized metabochemotherapy.

\section{Conclusions}

To the best of our knowledge, here we present for the first time that the upregulation of free PUFAs and etherPEs with PUFAs is a general trait of anthracyclins. According to literature, this increase is proferroptotic by promoting lipid peroxidation but other metabolic changes might counteract this type of cell death. This is the case of cholesterol lipids and saturated and monounsaturated glycerolipids, which are markers of the de novo lipogenesis in the LXR/SREBP pathways. PGs, which are negatively charged at physiological and pathophysiological pH, presented a strong increase in HepG2 and Huh7. This increase may play a key role in the higher uptake ratio of DOX of this cell lines. We found that these lipids showed disparate behavior depending on the cell type after the treatment with anthracyclins. These specificities partially explain the different sensitivity to anthracyclins by the effect of lipids on passive diffusion of the drugs, their metabolites, membrane fluidity, ER stress, and mechanism(s) of cell death. 
In conclusion, the behavior of the lipidome in the three cell lines suggests that: i) PUFAs or PUFAcontaining lipids may potentiate the effect of anthracyclins in a general way, and ii) statins may potentiate or attenuate the effect of anthracyclins in a cancer/patient specific way. Further research in animal models and patients is warranted to establish the possibility of transferring these observations from bench to bedside.

Funding: F.H. was funded by Svenska Sällskapet för Medicinsk Forskning (S17-0092) and Cancerfonden (201076PjF and CAN 2017/518). H.L. was funded by the Swedish Cancer Foundation (Cancerfonden, CAN2018/602), Swedish Research Council (2018-03301) and Swedish Research Council (2020-02367).

Acknowledgments: We are grateful to Dilruba Ahmed (Karolinska Institute, Sweden) for supplying Huh7 cell line.

Conflicts of Interest: The authors declare no conflict of interest.

\section{References}

(1) Cairns, R. A.; Harris, I. S.; Mak, T. W. Regulation of Cancer Cell Metabolism. Nat Rev Cancer 2011, 11 (2), 8595. https://doi.org/10.1038/nrc2981.

(2) Beloribi-Djefaflia, S.; Vasseur, S.; Guillaumond, F. Lipid Metabolic Reprogramming in Cancer Cells. Oncogenesis 2016, 5, e189. https://doi.org/10.1038/oncsis.2015.49.

(3) Kim; Cho; Yu; Jang; Yoon; Park; Cho. Comprehensive Metabolomic Search for Biomarkers to Differentiate Early Stage Hepatocellular Carcinoma from Cirrhosis. Cancers 2019, 11 (10), 1497. https://doi.org/10.3390/cancers11101497.

(4) Smith, M. R.; Chacko, B. K.; Johnson, M. S.; Benavides, G. A.; Uppal, K.; Go, Y.-M.; Jones, D. P.; Darley-Usmar, V. M. A Precision Medicine Approach to Defining the Impact of Doxorubicin on the Bioenergetic-Metabolite Interactome in Human Platelets. Redox Biology 2020, 28, 101311. https://doi.org/10.1016/j.redox.2019.101311.

(5) Sangineto, M.; Villani, R.; Cavallone, F.; Romano, A.; Loizzi, D.; Serviddio, G. Lipid Metabolism in Development and Progression of Hepatocellular Carcinoma. Cancers 2020, 12 (6), 1419. https://doi.org/10.3390/cancers12061419.

(6) Balgoma, D.; Pettersson, C.; Hedeland, M. Common Fatty Markers in Diseases with Dysregulated Lipogenesis. Trends in Endocrinology \& Metabolism 2019, 30 (5), 283-285. https://doi.org/10.1016/j.tem.2019.02.008.

(7) Wang, W.; Bai, L.; Li, W.; Cui, J. The Lipid Metabolic Landscape of Cancers and New Therapeutic Perspectives. Front. Oncol. 2020, 10, 605154. https://doi.org/10.3389/fonc.2020.605154.

(8) van der Zanden, S. Y.; Qiao, X.; Neefjes, J. New Insights into the Activities and Toxicities of the Old Anticancer Drug Doxorubicin. FEBS J 2020. https://doi.org/10.1111/febs.15583.

(9) Matyszewska, D.; Nazaruk, E.; Campbell, R. A. Interactions of Anticancer Drugs Doxorubicin and Idarubicin with Lipid Monolayers: New Insight into the Composition, Structure and Morphology. Journal of Colloid and Interface Science 2021, 581, 403-416. https://doi.org/10.1016/j.jcis.2020.07.092.

(10) Speelmans, G.; Staffhorst, R. W. H. M.; de Kruijff, B. The Anionic Phospholipid-Mediated Membrane Interaction of the Anti-Cancer Drug Doxorubicin Is Enhanced by Phosphatidylethanolamine Compared to Other Zwitterionic Phospholipids ${ }^{\dagger}$. Biochemistry 1997, $36 \quad$ (28), 8657-8662. https://doi.org/10.1021/bi963151g.

(11) Zugbi, S.; Winter, U.; Castañon, A.; Sampor, C.; Chantada, G.; Schaiquevich, P. Comparison of the Pharmacological Activity of Idarubicin and Doxorubicin for Retinoblastoma. Pediatr Blood Cancer 2019, 66 (1), e27441. https://doi.org/10.1002/pbc.27441.

(12) Todor, I. N.; Lukyanova, N. Y.; Chekhun, V. F. The Lipid Content of Cisplatin- and Doxorubicin-Resistant MCF7 Human Breast Cancer Cells. Exp Oncol 2012, 34 (2), 97-100. 
(13) Gewirtz, D. A Critical Evaluation of the Mechanisms of Action Proposed for the Antitumor Effects of the Anthracycline Antibiotics Adriamycin and Daunorubicin. Biochemical Pharmacology 1999, 57 (7), 727-741. https://doi.org/10.1016/S0006-2952(98)00307-4.

(14) Wander, D. P. A.; van der Zanden, S. Y.; van der Marel, G. A.; Overkleeft, H. S.; Neefjes, J.; Codée, J. D. C. Doxorubicin and Aclarubicin: Shuffling Anthracycline Glycans for Improved Anticancer Agents. J Med Chem 2020, 63 (21), 12814-12829. https://doi.org/10.1021/acs.jmedchem.0c01191.

(15) Gil-de-Gómez, L.; Balgoma, D.; Montero, O. Lipidomic-Based Advances in Diagnosis and Modulation of Immune Response to Cancer. Metabolites 2020, 10 (8). https://doi.org/10.3390/metabo10080332.

(16) Riad, A.; Bien, S.; Westermann, D.; Becher, P. M.; Loya, K.; Landmesser, U.; Kroemer, H. K.; Schultheiss, H. P.; Tschöpe, C. Pretreatment with Statin Attenuates the Cardiotoxicity of Doxorubicin in Mice. Cancer Res 2009, 69 (2), 695-699. https://doi.org/10.1158/0008-5472.CAN-08-3076.

(17) Boslem, E.; Weir, J. M.; Maclntosh, G.; Sue, N.; Cantley, J.; Meikle, P. J.; Biden, T. J. Alteration of Endoplasmic Reticulum Lipid Rafts Contributes to Lipotoxicity in Pancreatic $\beta$-Cells. Journal of Biological Chemistry 2013, 288 (37), 26569-26582. https://doi.org/10.1074/jbc.M113.489310.

(18) Zou, Y.; Henry, W. S.; Ricq, E. L.; Graham, E. T.; Phadnis, V. V.; Maretich, P.; Paradkar, S.; Boehnke, N.; Deik, A. A.; Reinhardt, F.; Eaton, J. K.; Ferguson, B.; Wang, W.; Fairman, J.; Keys, H. R.; Dančík, V.; Clish, C. B.; Clemons, P. A.; Hammond, P. T.; Boyer, L. A.; Weinberg, R. A.; Schreiber, S. L. Plasticity of Ether Lipids Promotes Ferroptosis Susceptibility and Evasion. Nature 2020, 585 (7826), 603-608. https://doi.org/10.1038/s41586-020-2732-8.

(19) Capes-Davis, A.; Theodosopoulos, G.; Atkin, I.; Drexler, H. G.; Kohara, A.; MacLeod, R. A. F.; Masters, J. R.; Nakamura, Y.; Reid, Y. A.; Reddel, R. R.; Freshney, R. I. Check Your Cultures! A List of Cross-Contaminated or Misidentified Cell Lines. Int J Cancer 2010, 127 (1), 1-8. https://doi.org/10.1002/ijc.25242.

(20) Kullenberg, Fredrik; Degerstedt, Oliver; Calitz, Carlemi; Pavlović, Nataša; Balgoma, David; Gråsjö, Johan; Sjögren, Erik; Hedeland, Mikael; Heindryckx, Femke; Lennernäs, Hans. In Vitro Cell Toxicity and Intracellular Uptake of Doxorubicin Exposed as a Solution or Liposomes: Implications for Treatment of Hepatocellular Carcinoma. Submitted.

(21) Balgoma, D.; Zelleroth, S.; Grönbladh, A.; Hallberg, M.; Pettersson, C.; Hedeland, M. Anabolic Androgenic Steroids Exert a Selective Remodeling of the Plasma Lipidome That Mirrors the Decrease of the de Novo Lipogenesis in the Liver. Metabolomics 2020, 16 (1), 12. https://doi.org/10.1007/s11306-019-1632-0.

(22) Betensky, R. A. The P-Value Requires Context, Not a Threshold. The American Statistician 2019, 73 (sup1), 115-117. https://doi.org/10.1080/00031305.2018.1529624.

(23) Wasserstein, R. L.; Lazar, N. A. The ASA's Statement on $p$-Values: Context, Process, and Purpose. The American Statistician 2016, 70 (2), 129-133. https://doi.org/10.1080/00031305.2016.1154108.

(24) Wasserstein, R. L.; Schirm, A. L.; Lazar, N. A. Moving to a World Beyond " $\mathrm{p}<0.05$ ". The American Statistician 2019, 73 (sup1), 1-19. https://doi.org/10.1080/00031305.2019.1583913.

(25) Gardner, M. J.; Altman, D. G. Confidence Intervals Rather than P Values: Estimation Rather than Hypothesis Testing. Br Med J (Clin Res Ed) 1986, 292 (6522), 746-750. https://doi.org/10.1136/bmj.292.6522.746.

(26) Balsinde, J. Roles of Various Phospholipases A2 in Providing Lysophospholipid Acceptors for Fatty Acid Phospholipid Incorporation and Remodelling. Biochem J 2002, 364 (Pt 3), 695-702. https://doi.org/10.1042/BJ20020142.

(27) Astudillo, A. M.; Balgoma, D.; Balboa, M. A.; Balsinde, J. Dynamics of Arachidonic Acid Mobilization by Inflammatory Cells. Biochim. Biophys. Acta 2012, $1821 \quad$ (2), 249-256. https://doi.org/10.1016/j.bbalip.2011.11.006.

(28) Poirier, Y.; Antonenkov, V. D.; Glumoff, T.; Hiltunen, J. K. Peroxisomal Beta-Oxidation--a Metabolic Pathway with Multiple Functions. Biochim Biophys Acta 2006, 1763 (12), 1413-1426. https://doi.org/10.1016/j.bbamcr.2006.08.034.

(29) Buranrat, B.; Suwannaloet, W.; Naowaboot, J. Simvastatin Potentiates Doxorubicin Activity against MCF-7 Breast Cancer Cells. Oncol Lett 2017. https://doi.org/10.3892/ol.2017.6783.

(30) Newell, M.; Patel, D.; Goruk, S.; Field, C. J. Docosahexaenoic Acid Incorporation Is Not Affected by Doxorubicin Chemotherapy in Either Whole Cell or Lipid Raft Phospholipids of Breast Cancer Cells in Vitro and Tumor Phospholipids in Vivo. Lipids 2020, 55 (5), 549-565. https://doi.org/10.1002/lipd.12252. 
(31) Achkar, I. W.; Kader, S.; Dib, S. S.; Junejo, K.; Al-Bader, S. B.; Hayat, S.; Bhagwat, A. M.; Rousset, X.; Wang, Y.; Viallet, J.; Suhre, K.; Halama, A. Metabolic Signatures of Tumor Responses to Doxorubicin Elucidated by Metabolic Profiling in Ovo. Metabolites 2020, 10 (7), 268. https://doi.org/10.3390/metabo10070268.

(32) Wang, B.; Tontonoz, P. Liver X Receptors in Lipid Signalling and Membrane Homeostasis. Nat Rev Endocrinol 2018, 14 (8), 452-463. https://doi.org/10.1038/s41574-018-0037-X.

(33) Lin, Y.-C.; Wang, J.-C.; Wu, M.-S.; Lin, Y.-F.; Chen, C.-R.; Chen, C.-Y.; Chen, K.-C.; Peng, C.-C. Nifedipine Exacerbates Lipogenesis in the Kidney via KIM-1, CD36, and SREBP Upregulation: Implications from an Animal Model for Human Study. IJMS 2020, 21 (12), 4359. https://doi.org/10.3390/ijms21124359.

(34) Monzel, J. V.; Budde, T.; Meyer zu Schwabedissen, H. E.; Schwebe, M.; Bien-Möller, S.; Lütjohann, D.; Kroemer, H. K.; Jedlitschky, G.; Grube, M. Doxorubicin Enhances Oxysterol Levels Resulting in a LXR-Mediated Upregulation of Cardiac Cholesterol Transporters. Biochemical Pharmacology 2017, 144, $108-119$. https://doi.org/10.1016/j.bcp.2017.08.008.

(35) Yun, U.-J.; Lee, J.-H.; Shim, J.; Yoon, K.; Goh, S.-H.; Yi, E. H.; Ye, S.-K.; Lee, J.-S.; Lee, H.; Park, J.; Lee, I. H.; Kim, Y.-N. Anti-Cancer Effect of Doxorubicin Is Mediated by Downregulation of HMG-Co A Reductase via Inhibition of EGFR/Src Pathway. Lab Invest 2019, 99 (8), 1157-1172. https://doi.org/10.1038/s41374-019-0193-1.

(36) Guillaumond, F.; Bidaut, G.; Ouaissi, M.; Servais, S.; Gouirand, V.; Olivares, O.; Lac, S.; Borge, L.; Roques, J.; Gayet, O.; Pinault, M.; Guimaraes, C.; Nigri, J.; Loncle, C.; Lavaut, M.-N.; Garcia, S.; Tailleux, A.; Staels, B.; Calvo, E.; Tomasini, R.; lovanna, J. L.; Vasseur, S. Cholesterol Uptake Disruption, in Association with Chemotherapy, Is a Promising Combined Metabolic Therapy for Pancreatic Adenocarcinoma. Proc Natl Acad Sci U S A 2015, 112 (8), 2473-2478. https://doi.org/10.1073/pnas.1421601112.

(37) Wanders, R. J. A. Peroxisomes, Lipid Metabolism, and Peroxisomal Disorders. Molecular Genetics and Metabolism 2004, 83 (1-2), 16-27. https://doi.org/10.1016/j.ymgme.2004.08.016.

(38) Dean, J. M.; Lodhi, I. J. Structural and Functional Roles of Ether Lipids. Protein Cell 2018, 9 (2), $196-206$. https://doi.org/10.1007/s13238-017-0423-5.

(39) Braverman, N. E.; Moser, A. B. Functions of Plasmalogen Lipids in Health and Disease. Biochimica et Biophysica Acta (BBA) - Molecular Basis of Disease 2012, 1822 (9), 1442-1452. https://doi.org/10.1016/j.bbadis.2012.05.008.

(40) Honsho, M.; Fujiki, Y. Plasmalogen Homeostasis - Regulation of Plasmalogen Biosynthesis and Its Physiological Consequence in Mammals. FEBS Lett 2017, 591 (18), 2720-2729. https://doi.org/10.1002/1873-3468.12743.

(41) Burdge, G. $\alpha$-Linolenic Acid Metabolism in Men and Women: Nutritional and Biological Implications: Current Opinion in Clinical Nutrition and Metabolic Care 2004, 7 (2), 137-144. https://doi.org/10.1097/00075197200403000-00006.

(42) Wallis, J. G.; Watts, J. L.; Browse, J. Polyunsaturated Fatty Acid Synthesis: What Will They Think of Next? Trends in Biochemical Sciences 2002, 27 (9), 467-473. https://doi.org/10.1016/S0968-0004(02)02168-0.

(43) Sprecher, H. Metabolism of Highly Unsaturated N-3 and n-6 Fatty Acids. Biochimica et Biophysica Acta (BBA) - Molecular and Cell Biology of Lipids 2000, 1486 (2-3), 219-231. https://doi.org/10.1016/S13881981(00)00077-9.

(44) Balgoma, D.; Astudillo, A. M.; Pérez-Chacón, G.; Montero, O.; Balboa, M. A.; Balsinde, J. Markers of Monocyte Activation Revealed by Lipidomic Profiling of Arachidonic Acid-Containing Phospholipids. J. Immunol. 2010, 184 (7), 3857-3865. https://doi.org/10.4049/jimmunol.0902883.

(45) Pérez-Chacón, G.; Astudillo, A. M.; Balgoma, D.; Balboa, M. A.; Balsinde, J. Control of Free Arachidonic Acid Levels by Phospholipases A2 and Lysophospholipid Acyltransferases. Biochimica et Biophysica Acta (BBA) Molecular and Cell Biology of Lipids 2009, 1791 (12), 1103-1113. https://doi.org/10.1016/j.bbalip.2009.08.007.

(46) Wanders, R. J. A.; Waterham, H. R.; Ferdinandusse, S. Metabolic Interplay between Peroxisomes and Other Subcellular Organelles Including Mitochondria and the Endoplasmic Reticulum. Front. Cell Dev. Biol. 2016, 3. https://doi.org/10.3389/fcell.2015.00083.

(47) Plumb, J. A.; Luo, W.; Kerr, D. J. Effect of Polyunsaturated Fatty Acids on the Drug Sensitivity of Human Tumour Cell Lines Resistant to Either Cisplatin or Doxorubicin. Br J Cancer 1993, 67 (4), 728-733. https://doi.org/10.1038/bjc.1993.133. 
bioRxiv preprint doi: https://doi.org/10.1101/2021.02.16.431377; this version posted February 17, 2021. The copyright holder for this preprint (which was not certified by peer review) is the author/funder. All rights reserved. No reuse allowed without permission.

(48) Kinsella, J. E.; Black, J. M. Effects of Polyunsaturated Fatty Acids on the Efficacy of Antineoplastic Agents toward L5178Y Lymphoma Cells. Biochem Pharmacol 1993, 45 (9), 1881-1887. https://doi.org/10.1016/0006-2952(93)90447-5.

(49) Germain, E.; Chajès, V.; Cognault, S.; Lhuillery, C.; Bougnoux, P. Enhancement of Doxorubicin Cytotoxicity by Polyunsaturated Fatty Acids in the Human Breast Tumor Cell Line MDA-MB-231: Relationship to Lipid Peroxidation. Int J Cancer 1998, 75 (4), 578-583. https://doi.org/10.1002/(sici)10970215(19980209)75:4<578::aid-ijc14>3.0.c0;2-5.

(50) Yang, W. S.; Kim, K. J.; Gaschler, M. M.; Patel, M.; Shchepinov, M. S.; Stockwell, B. R. Peroxidation of Polyunsaturated Fatty Acids by Lipoxygenases Drives Ferroptosis. Proc Natl Acad Sci USA 2016, 113 (34), E4966-E4975. https://doi.org/10.1073/pnas.1603244113.

(51) Koleini, N.; Nickel, B. E.; Edel, A. L.; Fandrich, R. R.; Ravandi, A.; Kardami, E. Oxidized Phospholipids in Doxorubicin-Induced Cardiotoxicity. Chem Biol Interact 2019, 303, 35-39. https://doi.org/10.1016/j.cbi.2019.01.032.

(52) Perez, M. A.; Magtanong, L.; Dixon, S. J.; Watts, J. L. Dietary Lipids Induce Ferroptosis in Caenorhabditiselegans and Human Cancer Cells. Developmental Cell 2020, 54 (4), 447-454.e4. https://doi.org/10.1016/j.devcel.2020.06.019.

(53) Zheng, J.; Conrad, M. The Metabolic Underpinnings of Ferroptosis. Cell Metab 2020, 32 (6), $920-937$. https://doi.org/10.1016/j.cmet.2020.10.011.

(54) Dixon, S. J.; Lemberg, K. M.; Lamprecht, M. R.; Skouta, R.; Zaitsev, E. M.; Gleason, C. E.; Patel, D. N.; Bauer, A. J.; Cantley, A. M.; Yang, W. S.; Morrison, B.; Stockwell, B. R. Ferroptosis: An Iron-Dependent Form of Nonapoptotic Cell Death. Cell 2012, 149 (5), 1060-1072. https://doi.org/10.1016/j.cell.2012.03.042.

(55) Stoffel, W.; Holz, B.; Jenke, B.; Binczek, E.; Günter, R. H.; Kiss, C.; Karakesisoglou, I.; Thevis, M.; Weber, A.-A.; Arnhold, S.; Addicks, K. $\triangle 6$-Desaturase (FADS2) Deficiency Unveils the Role of $\Omega 3$ - and $\Omega 6$-Polyunsaturated Fatty Acids. EMBO J 2008, 27 (17), 2281-2292. https://doi.org/10.1038/emboj.2008.156.

(56) Vriens, K.; Christen, S.; Parik, S.; Broekaert, D.; Yoshinaga, K.; Talebi, A.; Dehairs, J.; Escalona-Noguero, C.; Schmieder, R.; Cornfield, T.; Charlton, C.; Romero-Pérez, L.; Rossi, M.; Rinaldi, G.; Orth, M. F.; Boon, R.; Kerstens, A.; Kwan, S. Y.; Faubert, B.; Méndez-Lucas, A.; Kopitz, C. C.; Chen, T.; Fernandez-Garcia, J.; Duarte, J. A. G.; Schmitz, A. A.; Steigemann, P.; Najimi, M.; Hägebarth, A.; Van Ginderachter, J. A.; Sokal, E.; Gotoh, N.; Wong, K.-K.; Verfaillie, C.; Derua, R.; Munck, S.; Yuneva, M.; Beretta, L.; DeBerardinis, R. J.; Swinnen, J. V.; Hodson, L.; Cassiman, D.; Verslype, C.; Christian, S.; Grünewald, S.; Grünewald, T. G. P.; Fendt, S.-M. Evidence for an Alternative Fatty Acid Desaturation Pathway Increasing Cancer Plasticity. Nature 2019, 566 (7744), 403-406. https://doi.org/10.1038/s41586-019-0904-1.

(57) Stockwell, B. R.; Friedmann Angeli, J. P.; Bayir, H.; Bush, A. I.; Conrad, M.; Dixon, S. J.; Fulda, S.; Gascón, S.; Hatzios, S. K.; Kagan, V. E.; Noel, K.; Jiang, X.; Linkermann, A.; Murphy, M. E.; Overholtzer, M.; Oyagi, A.; Pagnussat, G. C.; Park, J.; Ran, Q.; Rosenfeld, C. S.; Salnikow, K.; Tang, D.; Torti, F. M.; Torti, S. V.; Toyokuni, S.; Woerpel, K. A.; Zhang, D. D. Ferroptosis: A Regulated Cell Death Nexus Linking Metabolism, Redox Biology, and Disease. Cell 2017, 171 (2), 273-285. https://doi.org/10.1016/j.cell.2017.09.021.

(58) Tachtsis, B.; Whitfield, J.; Hawley, J. A.; Hoffman, N. J. Omega-3 Polyunsaturated Fatty Acids Mitigate Palmitate-Induced Impairments in Skeletal Muscle Cell Viability and Differentiation. Front. Physiol. 2020, 11, 563. https://doi.org/10.3389/fphys.2020.00563.

(59) Ohta, M.; Kawano, H.; Notsu, T.; Naba, H.; Imada, K. Eicosapentaenoic Acid Attenuates Statin-Induced ER Stress and Toxicity in Myoblast. Biochemical and Biophysical Research Communications 2012, 424 (2), $301-$ 307. https://doi.org/10.1016/j.bbrc.2012.06.111.

(60) Chakraborty, S.; Doktorova, M.; Molugu, T. R.; Heberle, F. A.; Scott, H. L.; Dzikovski, B.; Nagao, M.; Stingaciu, L.-R.; Standaert, R. F.; Barrera, F. N.; Katsaras, J.; Khelashvili, G.; Brown, M. F.; Ashkar, R. How Cholesterol Stiffens Unsaturated Lipid Membranes. Proc Natl Acad Sci U S A 2020, 117 (36), 21896-21905. https://doi.org/10.1073/pnas.2004807117.

(61) Lee, Y.-S.; Lee, D.-H.; Choudry, H. A.; Bartlett, D. L.; Lee, Y. J. Ferroptosis-Induced Endoplasmic Reticulum Stress: Cross-Talk between Ferroptosis and Apoptosis. Mol Cancer Res 2018, 16 (7), 1073-1076. https://doi.org/10.1158/1541-7786.MCR-18-0055.

(62) Pavlović, N.; Calitz, C.; Thanapirom, K.; Mazza, G.; Rombouts, K.; Gerwins, P.; Heindryckx, F. Inhibiting IRE1 $\alpha-$ Endonuclease Activity Decreases Tumor Burden in a Mouse Model for Hepatocellular Carcinoma. Elife 2020, 9. https://doi.org/10.7554/eLife.55865. 
(63) Van Campenhout, S.; Tilleman, L.; Lefere, S.; Vandierendonck, A.; Raevens, S.; Verhelst, X.; Geerts, A.; Van Nieuwerburgh, F.; Van Vlierberghe, H.; Devisscher, L. Myeloid-Specific IRE1alpha Deletion Reduces Tumour Development in a Diabetic, Non-Alcoholic Steatohepatitis-Induced Hepatocellular Carcinoma Mouse Model. Metabolism 2020, 107, 154220. https://doi.org/10.1016/j.metabol.2020.154220.

(64) Salaroglio, I. C.; Panada, E.; Moiso, E.; Buondonno, I.; Provero, P.; Rubinstein, M.; Kopecka, J.; Riganti, C. PERK Induces Resistance to Cell Death Elicited by Endoplasmic Reticulum Stress and Chemotherapy. Mol Cancer 2017, 16 (1), 91. https://doi.org/10.1186/s12943-017-0657-0.

(65) Almanza, A.; Carlesso, A.; Chintha, C.; Creedican, S.; Doultsinos, D.; Leuzzi, B.; Luís, A.; McCarthy, N.; Montibeller, L.; More, S.; Papaioannou, A.; Püschel, F.; Sassano, M. L.; Skoko, J.; Agostinis, P.; de Belleroche, J.; Eriksson, L. A.; Fulda, S.; Gorman, A. M.; Healy, S.; Kozlov, A.; Muñoz-Pinedo, C.; Rehm, M.; Chevet, E.; Samali, A. Endoplasmic Reticulum Stress Signalling - from Basic Mechanisms to Clinical Applications. FEBS J 2019, 286 (2), 241-278. https://doi.org/10.1111/febs.14608.

(66) Dubbelboer, I. R.; Pavlovic, N.; Heindryckx, F.; Sjögren, E.; Lennernäs, H. Liver Cancer Cell Lines Treated with Doxorubicin under Normoxia and Hypoxia: Cell Viability and Oncologic Protein Profile. Cancers (Basel) 2019, 11 (7). https://doi.org/10.3390/cancers11071024

(67) Lages, E. B.; Fernandes, R. S.; Silva, J. de O.; de Souza, Â. M.; Cassali, G. D.; de Barros, A. L. B.; Miranda Ferreira, L. A. Co-Delivery of Doxorubicin, Docosahexaenoic Acid, and $\alpha$-Tocopherol Succinate by Nanostructured Lipid Carriers Has a Synergistic Effect to Enhance Antitumor Activity and Reduce Toxicity. Biomedicine \& Pharmacotherapy 2020, 132, 110876. https://doi.org/10.1016/j.biopha.2020.110876.

(68) Tadokoro, T.; Ikeda, M.; Ide, T.; Deguchi, H.; Ikeda, S.; Okabe, K.; Ishikita, A.; Matsushima, S.; Koumura, T.; Yamada, K.-I.; Imai, H.; Tsutsui, H. Mitochondria-Dependent Ferroptosis Plays a Pivotal Role in Doxorubicin Cardiotoxicity. JCl Insight 2020, 5 (9). https://doi.org/10.1172/jci.insight.132747.

(69) Serini, S.; Ottes Vasconcelos, R.; Nascimento Gomes, R.; Calviello, G. Protective Effects of $\omega-3$ PUFA in Anthracycline-Induced Cardiotoxicity: A Critical Review. IJMS 2017, 18 (12), 2689. https://doi.org/10.3390/ijms18122689.

(70) Varela-López, A.; Battino, M.; Navarro-Hortal, M. D.; Giampieri, F.; Forbes-Hernández, T. Y.; RomeroMárquez, J. M.; Collado, R.; Quiles, J. L. An Update on the Mechanisms Related to Cell Death and Toxicity of Doxorubicin and the Protective Role of Nutrients. Food and Chemical Toxicology 2019, 134, 110834. https://doi.org/10.1016/j.fct.2019.110834.

(71) Jakobisiak, M.; Golab, J. Statins Can Modulate Effectiveness of Antitumor Therapeutic Modalities: COMBINATIONS OF STATINS WITH OTHER DRUGS. Med. Res. Rev. 2010, 30 (1), 102-135. https://doi.org/10.1002/med.20162.

(72) Werner, M.; Sacher, J.; Hohenegger, M. Mutual Amplification of Apoptosis by Statin-Induced Mitochondrial Stress and Doxorubicin Toxicity in Human Rhabdomyosarcoma Cells: Statin-Induced Apoptosis in Rhabdomyosarcoma Cells. British Journal of Pharmacology 2004, 143 (6), 715-724. https://doi.org/10.1038/sj.bjp.0705928.

(73) Damrot, J.; Nübel, T.; Epe, B.; Roos, W. P.; Kaina, B.; Fritz, G. Lovastatin Protects Human Endothelial Cells from the Genotoxic and Cytotoxic Effects of the Anticancer Drugs Doxorubicin and Etoposide. British Journal of Pharmacology 2006, 149 (8), 988-997. https://doi.org/10.1038/sj.bjp.0706953.

(74) Henninger, C.; Huelsenbeck, J.; Huelsenbeck, S.; Grösch, S.; Schad, A.; Lackner, K. J.; Kaina, B.; Fritz, G. The Lipid Lowering Drug Lovastatin Protects against Doxorubicin-Induced Hepatotoxicity. Toxicology and Applied Pharmacology 2012, 261 (1), 66-73. https://doi.org/10.1016/j.taap.2012.03.012. 